\title{
Gene regulation gravitates towards either addition or multiplication when combining the effects of two signals
}

\author{
Authors: \\ Eric M. Sanford², Benjamin L. Emert ${ }^{2}$, Allison Coté $^{2}$, Arjun Raj ${ }^{1, \star}$ \\ ${ }^{1}$ Department of Bioengineering, School of Engineering and Applied Sciences, University of \\ Pennsylvania \\ ${ }^{2}$ Perelman School of Medicine, University of Pennsylvania \\ ${ }^{*}$ Correspondence to arjunrajlab@gmail.com
}

\begin{abstract}
Signals often ultimately affect the transcription of genes, and often, two different signals can affect the transcription of the same gene. In such cases, it is natural to ask how the combined transcriptional response compares to the individual responses. Mechanistic models can predict a range of combined responses, with the most commonly applied models predicting additive or multiplicative responses, but systematic genome-wide evaluation of these predictions are not available. Here, we performed a comprehensive analysis of the transcriptional response of human MCF-7 cells to two different signals (retinoic acid and TGF- $\beta$ ), applied individually and in combination. We found that the combined responses exhibited a range of behaviors, but clearly favored both additive and multiplicative combined transcriptional responses. We also performed paired chromatin accessibility measurements to measure putative transcription factor occupancy at regulatory elements near these genes. We found that increases in chromatin accessibility were largely additive, meaning that the combined accessibility response was the sum of the accessibility responses to each signal individually. We found some association between super-additivity of accessibility and multiplicative or super-multiplicative combined transcriptional responses, while sub-additivity of accessibility associated with additive transcriptional responses. Our findings suggest that mechanistic models of combined transcriptional regulation must be able to reproduce a range of behaviors.
\end{abstract}




\section{Introduction}

Suppose a cell at baseline expresses 100 copies of mRNA of gene X. If you give signal A, the cell expresses 200 copies of gene X. Give signal B, and you see 300 copies. What happens when you give both signals at the same time? Do the effects add (gene $X$ increases to 400 copies)? Multiply (600 copies)? Additive and to some extent multiplicative phenomenological models have seen widespread use due to their simple mechanistic basis. However, there is little systematic empirical evidence that either of these phenomenological models of combined responses are in general valid or should be favored in any way.

Part of the appeal of the additive and multiplicative phenomenological models is their emergence from simple and natural mechanistic models of transcriptional regulation. For instance, additive behavior naturally emerges from a model in which transcription factors can independently recruit polymerase to the promoter (Scholes et al. 2017; Bothma et al. 2015; Bender et al. 2012). Specifically, if signal A and signal B each induce the binding of different transcription factors to the enhancers of gene $X$, and these each independently result in an increased rate of binding of the polymerase to the promoter, then the total rate of binding would be the sum of the two independent contributions. (This additive prediction assumes that the binding events are not so frequent as to saturate the promoter.) Consistent with this behavior, the deletion of pairs of enhancers at the mouse $\beta$-globin locus resulted in additive reductions in gene expression (Bender et al., 2012), and CRISPRa-based activation of enhancer subsets resulted in additive increases in gene expression for several genes in an endometrial cancer cell line (Ginley-Hidinger et al., 2019). However, these experiments are typically limited to small sets of genes, making it difficult to conclude that additive behavior is the default, and indeed deviations from additive behavior are prevalent (Bothma et al., 2015; Ginley-Hidinger et al., 2019; Scholes et al., 2019).

Another oft-cited phenomenological observation is multiplicative integration of two transcriptional signals. One common model that can readily explain multiplicative integration is the so-called "thermodynamic model", in which it is assumed that equilibrium binding levels of RNA polymerase to the promoter is the control point for transcriptional regulation (Ackers et al., 1982; Bintu et al., 2005; Phillips et al., 2019; Scholes et al., 2017; Sherman and Cohen, 2012). In a simple instantiation with two transcription factors, A' and B', that mediate the effects of signals $A$ and $B$ on gene $X$, each factor individually lowers the binding energy of RNA polymerase to the promoter, increasing its affinity (Bintu et al., 2005). If both transcription factors are present, then the changes in binding energy add, and hence, given that the probability of a transcription factor recruiting RNA polymerase II depends exponentially on binding energy, the net change in equilibrium binding levels of RNA polymerase II would multiply. Multiplicative activation by two RNA polymerase-binding factors has been seen in mutant $\mathrm{E}$. coli experiments after $\lambda \mathrm{cl}$ and CRP binding sides were placed adjacent to a lac $Z$ promoter (Joung et al., 1994). In eukaryotes, thermodynamic models have been successful in predicting how engineered combinations of a few known transcription factor binding sequences next to a promoter affect the transcription of reporter genes in yeast and mouse embryonic stem cells, explaining $\sim 50 \%$ of the variance in reporter gene expression, and up to $72 \%$ of the variance when non-multiplicative interaction terms are included (Fiore and Cohen, 2016; Gertz et al., 2009). However, it is unclear from many of these assays, most of which 
focus on promoter manipulations, how prevalent and general the multiplicative predictions of the simplest version of the thermodynamic model are, especially given that many combined responses are known to follow more simple additive predictions.

While potential mechanisms underlying additive and multiplicative behavior are straightforward, there is no a priori reason to believe that most genes would follow one or the other, or either at all. Indeed, a larger class of "kinetic" models of transcription (which represent transcription as a coupled series of chemical reactions with distinct signal-responsive rates) have been shown to admit a wide variety of behaviors, ranging from sub-addition to super-multiplication (Scholes et al., 2017). A systematic test of these different phenomenological types of combined responses has yet to be done, in part because there is a lack of transcriptome-wide experiments in the literature that treat cells with two signals both individually and in combination. (A notable exception is (Goldstein et al., 2017), where the authors use dual-signal treatment and a heuristic approach to find synergistic and antagonistic genes but do not compare underlying phenomenological models of combined responses.) Thus, it remains unknown if combinatorial gene regulation is primarily additive, multiplicative, or a wide distribution of everything in between (and beyond).

Upstream of transcription, it is also unclear how multiple signals coordinately affect transcription factor binding activity at cis-regulatory elements. For instance, if each signal results in the binding of a specific set of transcription factors at a particular regulatory region individually, then do these two different sets of factors bind with the same probability when both signals are applied? Or are these probabilities affected by potential regulatory interactions between the signals? And how might these binding probabilities and potential interactions affect expression of the target genes? There is only limited transcription factor binding data available for experiments where cells receive multiple signals simultaneously (Goldstein et al., 2017), and then using ChIP-seq, which only reports binding profiles for specific transcription factors. Pairing combined response experiments with chromatin accessibility measurements, which correlate with aggregate transcription factor binding data (Thurman et al., 2012), has the potential to answer these questions in a more comprehensive manner than ChIP-seq would allow for.

Experimentally, part of what makes it difficult to compare phenomenological models of combined responses is that additive and multiplicative models can give nearly indistinguishable predictions, especially when one or both of the signals' effects are relatively small. As such, often experimental data will be consistent with, say, a multiplicative or additive model (or weighted variants of such models), but it is difficult to exclude the possibility of the other model, especially when only a limited number of genes are considered (Rothschild et al. 2014; Kaplan et al. 2008; Geva-Zatorsky et al. 2010; Rapakoulia et al. 2017). With current genome-wide expression profiling tools, however, it may be possible to query the integration modes of sufficiently many genes so as to discriminate between additive, multiplicative, and other phenomenological model predictions for at least some subset of genes, thus enabling a larger scale view of gene regulation's tendencies towards specific combined response behaviors.

Here, we profiled MCF-7 cells with paired RNA-seq and ATAC-seq measurements after we exposed them to retinoic acid, TGF- $\beta$, and both signals. We found that while genes' 
transcriptional responses exhibit a wide variety of behaviors when combining these two signals, they generally tended towards either addition or multiplication. ATAC-seq peaks, on the other hand, appeared to prefer addition as the default operation for combining two signal effects, although a minority of peaks clearly showed sub-additive or super-additive behavior. Genes with super-additive ATAC-seq peaks nearby were more likely to have a multiplicative or super-multiplicative transcriptional responses to retinoic acid and TGF- $\beta$. These data provide a comprehensive and systematic view of transcriptional responses to combined signal treatments.

\section{Results}

Upregulated genes gravitate toward addition and multiplication when combining the transcriptional effects of both signals

To quantitatively measure how gene regulation depends on multiple input signals, we performed three replicates of a paired RNA-seq and ATAC-seq experiment using MCF-7 cells (human breast carcinoma; selected for being well-characterized in its response to the two signals chosen). Prior to sequencing, we treated these cells with three different doses of TGF- $\beta$ $(1.25,5$, and $10 \mathrm{ng} / \mathrm{ml})$, retinoic acid $(50,200$, and $400 \mathrm{nM})$, or both signals (low, medium, and high dosages of both TGF- $\beta$ and retinoic acid simultaneously) for 72 hours (Figure 1B). We waited 72 hours to create a larger set of differentially expressed genes to use in subsequent analyses, and chose doses that led to broad changes in transcription and chromatin accessibility (Figure 1B; see methods for discussion of doses chosen). Initial analysis showed that the number of differentially expressed genes and differential peaks increased in a dose-dependent manner, and that all genes that were upregulated in both individual signal treatments were also upregulated in the combination treatment (Figure 1B). We focused our analysis on upregulated genes and upregulated ATAC-seq peaks due to their greater dynamic range in effect sizes and their more straightforward interpretation in the context of potential binding of activators to increase the transcription of nearby genes. (Note that our ethanol "vehicle" controls were performed at three different cell concentrations, and there were no significantly differentially expressed genes between concentrations. We did not, however, add the signals to different concentrations of cells or cells at different points in the cell cycle, in which context the signals may exert differential effects.)

We defined a master set of 1,398 genes by selecting the set of genes that were significantly upregulated in any dose of the combination treatment (log2 fold-change $\geq 0.5$ and Benjamini-Hochberg adjusted $p$ value $\leq 0.05$ ) and that had increased expression in all doses of each individual signal (Figure 1D). If we had selected the full set of all genes upregulated in any dose of the combined treatment, we would have analyzed a set of 2246 genes (Figure 1D). We required the change in expression to be positive for both individual signals, however, (i.e., $\Delta_{A}>$ 0 and $\Delta_{B}>0$ ) in order to maintain a consistent mapping between our categorical description of combined responses (e.g., "sub-additive", "super-multiplicative") and our continuous "c value" description of combined responses defined in Box 1 and Figure $1 \mathrm{~A}$. Requiring $\Delta_{A}>0$ and $\Delta_{B}>$ 0 in our master set of genes was necessary to guarantee that sub-additive combined transcriptional responses always had $c$ values less than 0 and that super-multiplicative responses always had $C$ values greater than 1 . Imposing the conditions of $\Delta_{A}>0$ and $\Delta_{B}>0$ 
removed $37.8 \%$ of the 2,246 genes that showed a significant increase in expression in the combined treatment (Figure 1D), leaving 1396 of the 1398 genes that ultimately fed into our analyses. Inclusion of genes with negative changes after individual signal treatments would require a more elaborate analysis framework to encompass the much larger variety of categorizations of potential responses that would be difficult to characterize with the number of genes in our analysis. (There were only two genes that were significantly downregulated in the combined treatment while also having $\Delta_{A}>0$ and $\Delta_{B}>0$ at all doses of each individual signal treatment; we elected to also include these two genes in our master set for the total of 1398.)

In our analysis of combined transcriptional responses, we assumed that retinoic acid and TGF-beta exhibited their effects on common target genes through distinct transcription factors. To justify this assumption, we confirmed that there was little cross-activation of pSMAD2 (which serves as a proxy for the readout of TGF-beta signaling) by performing immunofluorescence targeting pSMAD2 upon the addition of TGF-beta and retinoic acid individually (Supplemental Figure 4A-B). We saw that TGF-beta treatment rapidly increased the nuclear signal of pSMAD2 (by 40 minutes), which remained above baseline until the final time point at 72 hours, whereas retinoic acid treatment induced no changes in pSMAD2 signal relative to baseline (Supplemental Figure 4C-E). Nuclear expression of retinoic acid receptor alpha, which resides in the nucleus regardless of activation level (Mangelsdorf and Evans 1995), was stable between conditions at all time points (Supplemental Figure 5). Subsequent transcription factor motif analysis of our ATAC-seq data, however, suggested that retinoic acid receptor alpha (RARA) is activated by retinoic acid and not TGF-beta (See section titled "Motif analysis reveals that sub-additive peaks have a depletion of AP-1 and an enrichment of CTCF motifs while super-additive peaks have an enrichment of SMAD motifs"). This same motif analysis also suggested that retinoic acid and TGF-beta largely increased the activity of distinct transcription factors at the 72 hour time point, meaning that the secondary effects of retinoic acid and TGF-beta are likely mediated through the activity of distinct transcription factors.

Within our master set of 1,398 upregulated genes, we found a variety of different combined transcriptional response behaviors ranging from sub-addition to super-multiplication (Figure 1D-F). A transcriptional response is additive when the combined treatment effect represents the sum of the individual treatment effects, and multiplicative when the combined treatment represents the product of the individual treatment fold-changes. When both signals upregulate the expression of a gene, a multiplicative response is always higher than an additive response (Box 1; Figure 1A). To systematically classify the combined transcriptional responses at each gene, we used a statistical approach where we assumed each observation of a gene's expression value was derived from a Gaussian distribution (see methods). We classified a combined transcriptional response as sub-additive, additive, multiplicative, or super-multiplicative by comparing where a "perfect" hypothetical additive or multiplicative response lay with respect to the $80 \%$ confidence interval of the combined treatment's expression value (Supplemental Figure 7B). If both the hypothetical additive and the hypothetical multiplicative predictions lay within the confidence interval, we classified the response as ambiguous (Supplemental Figure 7B). Using this approach, we found that at the medium dose, $8.7 \%$ of genes had sub-additive combined transcriptional responses, $15.1 \%$ had additive responses, $2.1 \%$ had between an additive and multiplicative response, $11.7 \%$ had 
multiplicative responses, $18.7 \%$ had super-multiplicative responses, and $43.7 \%$ had ambiguous responses (Figure 1D), suggesting there is no single dominant category of combined response behavior. However, while the categories of addition and multiplication are appealing due to their correspondence to these simple phenomenological models, there is no a priori reason to believe that all or even most genes should necessarily adhere to either of these possibilities.

In order to quantitatively describe the combined transcriptional response characteristics of any gene without any presupposition of additive or multiplicative behavior, we defined a continuous parameter, hereby referred to as a gene's combined response factor or "c" value, that places the gene in an exact location on the spectrum of possible combined response behaviors (Box 1; Figure 1A). We could then solve for any gene's c value (within experimental error) after measuring the individual signal effects and the combined treatment effect. For an upregulated gene, a c value of 0 would indicate perfect addition, a c value of 1 indicates perfect multiplication, a $c$ value less than 0 indicates sub-addition, and a $c$ value greater than 1 indicates super-multiplication (see Figure $1 \mathrm{~A}$ for equation). We wondered what the distribution of $c$ values would look like across our master set of upregulated genes, and whether this distribution would tell us anything about genes' natural inclinations for specific combined response behaviors. For instance, if this distribution had its main peak at $c=0.5$, it would imply that genes naturally prefer to integrate two signals in a manner that lies between addition and multiplication. At all doses of combination treatment, we observed a wide peak centered around $c=0$ (additive), with a hint of a secondary peak at $c=1$ (multiplicative), suggesting that the integration of the effects of two signals is preferentially additive or multiplicative (Figure 1F; Supplemental Figure 6)).

In order to more rigorously demonstrate the preferences for these two values of c, we performed a series of simulations and statistical analyses. First, we generated simulated data taking into account measurement noise to estimate what the expected distributions of c would look like if signal integration was wholly additive or multiplicative. For each gene, we made three random draws for expression levels in both signal conditions based on the actual expression measurements and variance of those measurements to mimic our actual data (Supplemental Figure 7C). We then computed what we would have measured c to be based on these simulated measurements. This "null" produced broad peaks centered around $\mathrm{c}=0$ and $\mathrm{c}=1$, respectively, and a superposition of these two nulls appeared to match our experimentally measured distribution of $c$ values (Figure 1F). In order to more clearly demonstrate the existence of a secondary peak at $c=1$, we subtracted off from the distribution a purely additive null model (as computed above, fit to the observed distribution). The resultant residual distribution was a broad peak centered roughly around $c=1$ (a Gaussian fit to the residual gave a fit centered at $\mathrm{c}=1.12$ and $\mathrm{c}=1.00$ at medium and high doses, respectively), consistent with our multiplicative simulated data (Figure 1F; Supplemental Figure 6A). We showed that this residual distribution was not likely to be due to statistical fluctuations by computing a $p$-value for the possibility of obtaining as big a residual in a sliding window by random chance (Supplemental Figure 6B). Overall, while there is the possibility of further peaks within our data, our data most strongly support the existence of two peaks in the c-value histogram, one corresponding most closely with an additive model, and the other with a multiplicative model. 
While our superimposed distribution of $\mathrm{c}$ values derived from simulated additive and multiplicative combined responses bears a close resemblance to our observed distribution of $c$ values in the neighborhoods of $c=0$ (addition) and $c=1$ (multiplication), the tails of the observed $c$ value distribution are clearly heavier (Figure 1F). These heavier tails illustrate that biological variation, rather than measurement error, produces a significant amount of sub-additive $(c<0)$ and super-multiplicative $(c>1)$ combined transcriptional responses.

We next wondered how a gene's combined response factor (c value) depended on dosage of the input signals. In theory, the $c$ value might remain stable as dosage increases, monotonically increase or decrease as dosage increases, or may appear to be "random" with respect to dose, perhaps due to complex unobserved dose-dependent gene regulatory interactions. To distinguish between these possibilities, we plotted how a set of upregulated genes' $c$ values changed as they moved from low to medium to high dose of combination treatment with retinoic acid and TGF- $\beta$ (Supplemental Figure 1B-D). To generate a subset of reliable $\mathrm{c}$ value estimates within our master set of genes, we selected genes for which $\frac{\Delta_{A} \Delta_{B}}{x_{\text {baseline }}} \geq$ 2 transcripts per million (TPM) and $\frac{\Delta_{A} \Delta_{B}}{x_{\text {baseline }}} \geq x_{\text {baseline }}$ (Supplemental Figure 1C). Since $\frac{\Delta_{A} \Delta_{B}}{x_{\text {baseline }}}$ captures the difference between the multiplicative and additive predictions, the estimation of $c$ is more reliable when $\frac{\Delta_{A} \Delta_{B}}{x_{\text {baseline }}}$ is large, because when that number is large it is less susceptible to technical variability. We found that most genes' c values were stable or moderately decreased with increasing signal dose, suggesting that the function a gene uses to combine two signals is mostly stable, with a tendency towards "saturation" with increasing dose (i.e., the function itself moves in the direction of sub-additivity when dosage increases).

\section{Increases in chromatin accessibility are largely additive}

Transcriptional regulation is thought to occur largely via the binding of transcription factors, but it remains unknown how the transcription factors associated with the effects of individual signals might interact upon the addition of both signals simultaneously. We performed ATAC-seq on the same populations described earlier, reasoning that the observation that changes in chromatin accessibility have been shown to correlate with changes in aggregate transcription factor binding activity (Thurman et al., 2012) meant that we could infer something about transcription factor binding at these sites. Note that the extent to which changes in chromatin accessibility quantitatively reflect changes in transcription factor occupancy is currently unknown, and may depend on the mechanism by which binding of transcription factors leads to opening of chromatin, such as displacement of nucleosomes by pioneer factors, recruitment of secondary transcription factors, or recruitment of chromatin remodeling complexes (Zaret and Carroll 2011; Klemm et al. 2019). Reassuringly, our initial motif enrichment analysis revealed that retinoic acid receptor alpha (RARA) and three TGF- $\beta$ pathway transcription factor motifs (SMAD3, SMAD4, and SMAD9) were highly enriched in their respective individual signal treatment conditions (Supplemental Figure 2B). Note that our motif analysis also indicated some degree of activation of RARA by TGF- $\beta$ and some degree of activation of SMAD3 and SMAD9 by retinoic acid, which led to even higher enrichment levels of these factors in the combined treatment condition (Supplemental Figure 2A). We did not, 
however, observe cross-activation of pSMAD2 by retinoic acid in immunofluorescence experiments (Supplemental Figure 4).

We then wondered how well simple additive and multiplicative phenomenological predictions corresponded to the increase in chromatin accessibility at upregulated peaks in the combined treatment. We found that an additive model was generally highly predictive and matched the observed increases in ATAC-seq fragment counts more accurately than the multiplicative model; the multiplicative model generally predicted larger changes in accessibility than we experimentally observed (Supplemental Figure 3). To quantify the degree to which the additive prediction was accurate, we defined a new metric, the fold-change difference in accessibility from an additive model prediction, hereby referred to as a peak's "d" value, to create a distribution that illustrates the extent to which the size of a peak in the combination treatment condition deviated from additive model predictions (Figure $2 \mathrm{~A}-\mathrm{B}$ ). We found that at upregulated peaks, our observed distribution of $d$ values was centered at zero, highlighting how addition appears to be the "default" operation at upregulated peaks (Figure 2C). This default additive behavior may correspond to a mechanistic model in which each signal stimulates an independent set of chromatin-opening transcription factors that independently and rarely bind DNA (Figure 2E).

Given the general accuracy of the additive model for upregulated peaks, we wondered to what extent deviations from additive model predictions represented true deviations as opposed to just measurement error. We produced randomly generated simulated data that matched the statistical properties of our actual data, assuming that the combined treatment would result on average in perfectly additive peak sizes (see methods for details). We found that our observed data are more widely dispersed than the simulations, indicating that a fair number of peaks are significantly sub-additive or super-additive (Figure 2C). We found that $19 \%$ of peaks were sub-additive and $16 \%$ of peaks were super-additive when we considered additive peaks to be those where a perfectly additive prediction lied within the $80 \%$ confidence interval of the measured peak fragment counts (Figure 2D). Thus, most upregulated ATAC-seq peaks displayed additive or near-additive combined responses, but significant fractions of peaks also displayed both sub-additive and super-additive combined responses.

Super-additive peaks and pairs of individual signal-dominant peaks are more likely to be found near genes with multiplicative transcriptional responses

We next wondered if we could uncover the patterns of cis-regulatory element activity that may dictate how a gene's regulatory behavior would encode the observed integration of the transcriptional effects of two signals. We reasoned that the number of upregulated ATAC-seq peaks near a gene or the manner in which the nearby peaks themselves integrated the two signals' effects may predict the gene's combined transcriptional response behavior. For each transcriptionally upregulated gene, we counted the number of sub-additive, additive, and super-additive ATAC-seq peaks within $100 \mathrm{~kb}$ of its transcription start site. We found that, on average, genes that were transcriptionally additive had 2.7x more sub-additive ATAC-seq peaks nearby than genes with multiplicative transcriptional responses (medium dose, $\mathrm{p}=$ 0.0012 ). Genes with multiplicative and super-multiplicative transcriptional responses had $2.5 x$ or 2.6x, respectively, more super-additive ATAC-seq peaks nearby than genes with additive 
transcriptional responses (Figure $3 A$, medium dose, $p=0.0016$ or $p=0.00016$, respectively). Genes with multiplicative transcriptional responses also had more additive ATAC-seq peaks nearby than every other combined transcriptional response behavior at each dose we tested, with 1.3x more additive peaks than genes with additive transcriptional responses (Figure 3A, medium dose, $p=0.12$ compared to additive transcriptional responses, $p=0.00089$ compared to ambiguous transcriptional responses). The most prominent effect in this analysis was the observation that super-additive peaks are more likely to be near genes with multiplicative and super-multiplicative transcriptional responses, suggesting that cooperative interactions between transcription factors at neighboring enhancers may increase the expression of a gene when both signals are added together; i.e., the gene's combined response factor.

When both signals affect accessibility at the same region of DNA, interactions between each signal's induced transcription factors and associated complexes can make it difficult to discriminate between mechanistic models of how transcription factors interact to regulate transcription. However, if the transcription factors affected by retinoic acid or TGF- $\beta$ bind to distinct regions of DNA around the same gene, then there are likely no interactions between induced transcription factors and one can in principle discriminate between a simple thermodynamic model (prediction: multiplicative transcriptional effects) and an independent recruitment model (prediction: additive transcriptional effects). To increase the likelihood of selecting retinoic acid and TGF- $\beta$-exclusive transcription factor binding events, we searched near genes for upregulated peaks that responded exclusively to either retinoic acid or TGF- $\beta$. (We defined "exclusive" here to mean that the peak size increase for a single signal was $\geq 90 \%$ that of the sum of the absolute peak size changes from both individual signals. Note that to generate a sufficiently large sample, we had to allow the selected genes to have non-exclusive peaks nearby as well because only $8.0 \%$ of gene-adjacent differential peaks met this exclusivity criteria for retinoic acid and only $3.4 \%$ met this criteria for TGF- $\beta$.) We then considered how likely genes with different combined transcriptional response behaviors were to have at least one retinoic acid-dominant and one TGF- $\beta$-dominant peak nearby $(<100 \mathrm{~kb}$ to the transcription start site). We found that at each dose, genes with multiplicative transcriptional responses were the most likely to have at least one retinoic-acid-dominant and one TGF- $\beta$-dominant upregulated peak nearby (Figure 3B; $2.4 \mathrm{x}$ increase compared to genes with additive transcriptional responses at high dose, $p=0.044$ ), suggesting that the effects of independently-upregulated peaks are most likely to act together to multiplicatively regulate transcription, which is more consistent with the predictions of the thermodynamic model.

Motif analysis reveals that sub-additive peaks have a depletion of AP-1 and an enrichment of CTCF motifs while super-additive peaks have an enrichment of SMAD motifs

We next wondered if the activity of particular transcription factors was associated with combined increases in chromatin accessibility that were either sub-additive, additive, or super-additive. To approach this question, we first identified a set of the 50 transcription factors with the largest predicted changes in activity in our full set of differential peaks using the chromVAR package and its associated curated cisBP database of transcription factor motifs (Schep et al., 2017). These factors included the canonical retinoic acid and TGF- $\beta$ effectors RARA, SMAD3, SMAD4, and SMAD9, as well as forkhead box factors and ETS family 
factors (enriched in the retinoic acid condition), AP-1 factors (enriched in the TGF- $\beta$ condition), and HOX and NF-k $\beta$ factors (enriched in both the retinoic acid and TGF- $\beta$ conditions). We manually added the CTCF motif to this set of enriched motifs to see if putative insulators behaved differently than other cis-regulatory elements. For each of these transcription factors, we calculated a motif enrichment score in each condition (based on the bias-uncorrected deviation score from chromVAR) that represents the percentage change in ATAC-seq fragment counts in all peaks that contain the given transcription factor's motif (Figure 4A). For example, the motif enrichment score of 0.19 for RARA in the retinoic acid condition means that peaks containing RARA motifs saw an average increase of $19 \%$ in ATAC-seq fragment counts after retinoic acid treatment (note that to decrease the variability of motif enrichment score estimates, we pooled together the low, medium, and high doses for each condition). Retinoic acid and TGF- $\beta$ treatment thus led to activation of both distinct and shared transcription factor families, with combination treatment showing similar activation of distinct factors and higher activation of shared factors (Figure 4A).

We then tested if any of the transcription factor motifs we identified were more enriched in sub-additive or super-additive peaks compared to additive peaks. Because sub-additive peaks were on average $8 \%$ narrower and super-additive peaks were on average $36 \%$ wider than additive peaks (Figure 4B), we compared the number of motif matches found per $150 \mathrm{bp}$ of each peak type. When compared to additive peaks, sub-additive peaks showed $21 \%$ fewer total motif matches per $150 \mathrm{bp}$ in our set of enriched motifs $\left(p=6.6 \mathrm{e}^{-14}\right)$ and $7 \%$ fewer total motif matches per 150 bp when using the entire cisBP database $\left(p=1.5 e^{-8}\right)$, suggesting that sub-additive peaks are slightly depleted for motifs overall while being even more depleted for the motifs in our enriched set (Figure 4C). Sub-additive peaks were especially depleted for SMARCC 1 motifs $\left(0.6 x\right.$ the motif density of additive peaks, $\left.p=1.2 e^{-15}\right)$ as well as $A P-1$ subunit motifs such as JUN ( $0.6 x$ density, $\left.p=3.4 e^{-13}\right)$ and FOS $\left(0.6 x\right.$ density, $p=6.2 e^{-13}$; Figure $\left.4 E\right)$. Sub-additive peaks did, however, show a strong enrichment of CTCF motifs, with 1.6x and $3.2 x$ more motif matches per 150 bp than in additive and super-additive peaks, respectively ( $p$ $=2.9 \mathrm{e}^{-11}$ and $\mathrm{p}<2.2 \mathrm{e}^{-16}$, respectively; Figure $4 \mathrm{E}$ ), suggesting that insulator proteins like CTCF may attenuate the combined activity of signal-induced transcription factors or the chromatin remodeling complexes they may recruit.

Super-additive peaks generally had the same motif densities as additive peaks, with the exception of an increase in the density of SMAD motifs $(1.8 \mathrm{x}, 1.4 \mathrm{x}$, and $1.5 \mathrm{x}$ increase of SMAD3, SMAD4, and SMAD9 motif density compared to additive peaks; $p=4.4 \mathrm{e}-4, p=8.5 \mathrm{e}^{-5}$, $\left.p=1.5 e^{-5}\right)$ and a depletion of several ETS family factors $(0.6 x$ the motif density of additive peaks for ELF1, $p=0.048$; Figure $4 E$ ). The higher frequency of SMAD motifs in super-additive peaks suggests that SMAD transcription factors may interact with retinoic acid-induced chromatin remodeling factors or retinoic acid-induced transcription factors.

We next wondered how strong of an effect each motif had on its "host" peak's tendency to have a super- or sub-additive combined response. To estimate this effect, we took each motif, found all peaks that contained that motif that were upregulated by both TGF- $\beta$ and retinoic acid individually, and computed the deviation from the additive prediction ( $d$ value) (Figure 4F). Here, we found that the presence of SMAD or NF-K $\beta$ motifs resulted in the largest increases in a peak's tendency to be super-additive, possibly suggesting that SMAD proteins 
have one of the most potent interactions with a retinoic acid-induced transcription factor or chromatin remodeling complex in our system. Note that since we observed that both retinoic acid and TGF- $\beta$ led to increases in NF-K $\beta$ factor activity (Figure 4A), the increase in $d$ value associated with NF-K $\beta$ motifs' could reflect synergistic activation of NF- $\mathrm{k} \beta$ factors rather than cooperative interactions between NF- $\mathrm{k} \beta$ factors and other induced transcription or chromatin remodeling factors.

We hypothesized that cooperative interactions between transcription factors may lead to super-additive increases in chromatin accessibility. To evaluate if our data supported this hypothesis, we tested if super-additive peaks were more likely to have both a retinoic acid-enriched motif and a TGF- $\beta$-enriched motif. We defined retinoic acid-enriched factors to be retinoic acid receptor, FOX, and ETS-family factors, and we defined TGF- $\beta$-enriched motifs to be SMAD, AP-1, BACH, BATF, SMARCC1, NFE2, NFE2L2, MAFF, and MAFK factors. We found that all categories of peaks (including super-additive) were less likely to have dual-motifs than expected based on a null distribution we generated by randomly shuffling motif matches across peaks (Figure 4D, $p<0.001$ for sub-additive, additive, and super-additive peaks, see methods for null distribution details. The higher expected rates of dual motif matches may be explained by the fact that binding sites for the same transcription factor are often found in clusters (Gotea et al., 2010); the motif shuffling process disperses these binding sites more evenly). Super-additive peaks were closer to their higher expected rate than sub-additive and additive peaks (with super-additive, additive, and sub-additive peaks having dual-motif match rates that were $10 \%, 21 \%$, and $27 \%$ lower than expected, respectively). While the effect is modest, the relatively higher rate of dual-motif matches in super-additive peaks provides some support for the idea that peak super-additivity may result from cooperative interactions between retinoic acid and TGF- $\beta$ transcriptional effectors.

\section{Discussion}

Here, we have asked how cells respond transcriptionally to combinations of signals. In principle, the transcriptional response to such combinations could range over a spectrum of different possibilities, and the mechanistically-motivated "additive" and "multiplicative" modes need not be favored. We were thus surprised to see that combined responses did seem to favor the simple additive and multiplicative phenomenological models.

Additive and multiplicative outcomes need not in principle be favored in any way. Mechanistic models of transcriptional regulation, in particular kinetic models, can yield a range of phenomenological predictions, spanning these two possibilities and more (Scholes et al., 2017). The primary reason behind the popularity of the independent recruitment model (which predicts additive behavior) and the thermodynamic model (which predicts multiplicative behavior) is their simplicity, hence our surprise. It is of course important to realize that just because the predictions of a particular mechanistic model match these experimental outcomes does not mean that there are not other models that may also match our experimental findings. Indeed, these simple models, which inherently posit that regulation acts via a single rate-limiting step, are incompatible with recent results demonstrating that regulation can act via multiple steps, and also typically have not been applied to complex regulatory mechanisms that involve long-range promoter-enhancer contacts (Bartman et al., 2019; Blau et al., 1996; 
Fuda et al., 2009; Nechaev and Adelman, 2011; Stampfel et al., 2015). Further combined theoretical and experimental work would be required to determine the experimental signatures beyond simple additivity or multiplicativity that could distinguish such models from each other.

Although they were the minority of cases, we did observe a large number of sub-additive and super-multiplicative combined responses. Super-multiplicative combined responses may reflect cooperative interactions between retinoic acid and TGF- $\beta$ induced factors, in which binding of a retinoic acid factor to DNA strengthens the binding of a TGF- $\beta$ factor to nearby DNA or vice versa. This type of interaction is consistent with our finding that super-multiplicative gene expression responses are associated with nearby super-additive ATAC-seq peaks (on the assumption that super-additivity of ATAC-seq peaks reflects cooperative binding of transcription factors to DNA) (Figure 3A). However, given that ATAC-seq peaks likely have additional routes to super-additive increases in accessibility (perhaps involving chromatin remodeling factors affected by our signals), further work would be needed to demonstrate that super-multiplicative transcriptional responses are indeed a result of direct binding interactions at enhancers. Sub-additive transcriptional responses have been proposed to reflect saturation of cis-regulatory elements (Bothma et al., 2015; Scholes et al., 2019). Saturated cis-regulatory elements would in principle show up as sub-additive ATAC-seq peaks in our analysis, but we did not observe an increase in sub-additive peaks near genes with sub-additive combined responses (with the exception of a small increase at high dose; Figure $3 A)$. This lack of association suggests that saturation of DNA binding sites may not be sufficient to explain sub-additive combined transcriptional responses; instead, the sub-additive behavior may be a property specifically encoded through the interactions between regulatory factors. It could also be that chromatin accessibility does not quantitatively reflect saturating transcription factor binding.

Our combined transcriptional responses were measured using bulk RNA-sequencing, which averages the transcriptional effects of retinoic acid and TGF- $\beta$ across millions of cells. Heterogeneity in the response of individual cells could mean that what we observed, for instance, as a multiplicative transcriptional response at the population level is actually a mixture of sub and super-multiplicative transcriptional responses at the single-cell level. Future studies might combine microfluidic delivery of cell signals with live imaging of transcription to measure the response to both individual and combined signal treatments in the same single cells, thereby revealing the extent to which the combined response factor for a given gene displays cell-to-cell heterogeneity (Zhang et al. 2019). High amounts of heterogeneity could suggest a need for even greater flexibility in biophysical models of combined transcriptional responses.

In our dataset, the combined response factor remained largely constant over a range of doses. This constancy suggests that whatever the functional interaction is between the factors responsible for the particular mode of combined response, that interaction is quantitatively maintained through doses (with some evidence for saturation at high dose). Such behavior may constrain potential models for interactions, because in principle the interactions could be highly dose dependent. Another open question is whether the mode of combined response for a particular gene depends on the particular signals applied or contextual factors that may vary between cell lines. Further studies may reveal these dependencies. 
Another interesting feature of our data was the general lack of strong correspondence between changes in chromatin accessibility and changes in transcriptional output. While we were able to identify some trends, we could not find any strict rules for e.g. what transcription factors associated with what types of combined responses. We found this lack of correspondence surprising, given that transcription factors are the dominant form of transcriptional regulation. There are many potential explanations for this observation. One is that the degree of chromatin accessibility is not as correlated with aggregate transcription factor occupancy levels as we expected. For instance, it may be that accessibility may only change for some types of transcription factor-DNA interactions and not others. Another possibility is that our analysis does not take into account precisely which peaks near a given gene correspond to regulatory elements and which ones do not. This mapping remains largely unknown, although information about what pieces of chromatin spatially contact which other ones may help narrow down the choices (Fulco et al., 2019; Jin et al., 2013; Rao et al., 2014; Ruf et al., 2011). Finally, it is also simply possible that the rules governing transcriptional output are highly complex and thus not straightforward to discern from the analyses we performed. In particular, it could be that the genome sequence itself is simply too limited to provide enough sampling of the possible configuration space of transcription factor binding motifs to extract rules. The use of massively parallel reporter assays (Kwasnieski et al., 2014; Patwardhan et al., 2012) or similar synthetic approaches (Bogard et al., 2019; Rosenberg et al., 2015) may help reveal such rules. 


\section{Materials and Methods}

\begin{tabular}{|c|c|c|c|c|}
\hline \multicolumn{5}{|c|}{ Key Resources Table } \\
\hline $\begin{array}{l}\text { Reagent type } \\
\text { (species) or } \\
\text { resource }\end{array}$ & Designation & $\begin{array}{l}\text { Source or } \\
\text { reference }\end{array}$ & Identifiers & $\begin{array}{l}\text { Additional } \\
\text { information }\end{array}$ \\
\hline $\begin{array}{l}\text { cell line (Homo } \\
\text { sapiens) }\end{array}$ & $\begin{array}{l}\text { MCF-7 (breast } \\
\text { carcinoma) }\end{array}$ & ATCC & $\begin{array}{l}\text { ATCC } \\
\text { HTB-22, } \\
\text { lot } \\
64125078\end{array}$ & \\
\hline $\begin{array}{l}\text { Peptide, } \\
\text { recombinant } \\
\text { protein }\end{array}$ & TGF-beta & Sigma & T7039 & \\
\hline $\begin{array}{l}\text { chemical } \\
\text { compound, drug }\end{array}$ & $\begin{array}{l}\text { all trans } \\
\text { retinoic acid }\end{array}$ & Sigma & R2625 & \\
\hline $\begin{array}{l}\text { cell culture } \\
\text { reagent }\end{array}$ & $\begin{array}{l}\text { Charcoal- } \\
\text { stripped FBS }\end{array}$ & Gemini & $100-119$ & \\
\hline $\begin{array}{l}\text { commercial assay } \\
\text { or kit }\end{array}$ & $\begin{array}{l}\text { miRNeasy RNA } \\
\text { extraction kit }\end{array}$ & Qiagen & 217004 & \\
\hline $\begin{array}{l}\text { commercial assay } \\
\text { or kit }\end{array}$ & $\begin{array}{l}\text { NEBNext } \\
\text { Poly(A) mRNA } \\
\text { Magnetic } \\
\text { Isolation } \\
\text { Module }\end{array}$ & $\begin{array}{l}\text { New } \\
\text { England } \\
\text { Biolabs }\end{array}$ & E7490 & \\
\hline $\begin{array}{l}\text { commercial assay } \\
\text { or kit }\end{array}$ & $\begin{array}{l}\text { NEBNext Ultra } \\
\text { II RNA Library } \\
\text { Prep Kit for } \\
\text { Illumina }\end{array}$ & $\begin{array}{l}\text { New } \\
\text { England } \\
\text { Biolabs }\end{array}$ & E7770 & \\
\hline $\begin{array}{l}\text { Sequence- } \\
\text { based reagent }\end{array}$ & $\begin{array}{l}\text { NEBNext } \\
\text { Multiplex } \\
\text { Oligos for } \\
\text { Illumina }\end{array}$ & $\begin{array}{l}\text { New } \\
\text { England } \\
\text { Biolabs }\end{array}$ & E7600 & \\
\hline
\end{tabular}




\begin{tabular}{|c|c|c|c|c|}
\hline $\begin{array}{l}\text { commercial assay } \\
\text { or kit }\end{array}$ & $\begin{array}{l}\text { Tagment DNA } \\
\text { Enzyme and } \\
\text { Buffer }\end{array}$ & Illumina & 20034197 & \\
\hline $\begin{array}{l}\text { Sequence- } \\
\text { based reagent }\end{array}$ & $\begin{array}{l}\text { ATAC-seq } \\
\text { indices } \\
\text { (custom } \\
\text { oligos) }\end{array}$ & $\begin{array}{l}\text { Integrated } \\
\text { DNA } \\
\text { Technologie } \\
\text { s }\end{array}$ & & $\begin{array}{l}\text { See } \\
\text { (Buenrostro et } \\
\text { al. 2013) for } \\
\text { custom index } \\
\text { sequences }\end{array}$ \\
\hline antibody & $\begin{array}{l}\text { Rabbit } \\
\text { anti-human } \\
\text { RARA }\end{array}$ & Sigma & HPA058282 & \\
\hline antibody & $\begin{array}{l}\text { Rabbit } \\
\text { anti-human } \\
\text { pSMAD2 }\end{array}$ & $\begin{array}{l}\text { Cell } \\
\text { Signaling } \\
\text { Technology }\end{array}$ & $18338 \mathrm{~T}$ & \\
\hline antibody & $\begin{array}{l}\text { Goat } \\
\text { anti-rabbit } \\
\text { lgG, Alexa } \\
\text { Fluor } 647\end{array}$ & $\begin{array}{l}\text { Thermo } \\
\text { Fisher } \\
\text { Scientific }\end{array}$ & A-21244 & \\
\hline
\end{tabular}

Cell culture and signal delivery

We acquired one vial of MCF-7 cells from ATCC (lot 64125078), which we expanded in DMEM/F12 with 5\% FBS and 1\% penicillin/streptomycin. Prior to adding retinoic acid and TGF- $\beta$, the cells experienced a total of 13 passages and one freeze/thaw cycle. Because normal FBS can have significant amounts of retinoic acid (Napoli, 1986), we cultured the cells in a modified medium containing charcoal-stripped FBS, with each batch consisting of $50 \mathrm{ml}$ charcoal-stripped FBS (Gemini, 100-119), $5 \mathrm{ml}$ penicillin/streptomycin (Invitrogen, 15140-122), and $500 \mathrm{ml}$ DMEM/F12 (Gibco, 10565018). We grew the MCF-7 cells in this charcoal-stripped FBS-containing medium for a total of 70 or 71 days prior to treating them with retinoic acid and TGF- $\beta$. Our MCF-7 cells were negative for mycoplasma contamination after all RNA and ATAC sequencing experiments.

For our dose-response experiment, we split two $80 \%$ confluent $10 \mathrm{~cm}$ dishes equally into 12 different $10 \mathrm{~cm}$ dishes, and waited 24 hours prior to adding media containing retinoic acid (Sigma, R2625), TGF- $\beta$ (Sigma, T7039), or both signals. Because the cells grew faster when exposed to retinoic acid and slower when exposed to TGF- $\beta$, we included two additional control conditions that had $50 \%$ and $150 \%$ of the starting cell density to test for potential cell-density effects (these additional conditions covered the range of cell-densities seen at the endpoint of our experiments). We treated cells for 72 hours in three doses of retinoic acid 
(50nM, $200 \mathrm{nM}$, and $400 \mathrm{nM})$, TGF- $\beta(1.25 \mathrm{ng} / \mathrm{ml}, 5 \mathrm{ng} / \mathrm{ml}, 10 \mathrm{ng} / \mathrm{ml})$, or both signals (50 nM retinoic acid $+1.25 \mathrm{ng} / \mathrm{ml}$ TGF- $\beta, 200 \mathrm{nM}$ RA $+5 \mathrm{ng} / \mathrm{ml}$ TGF- $\beta, 400 \mathrm{nM}$ RA $+10 \mathrm{ng} / \mathrm{ml}$ TGF- $\beta$ ). The medium dose we chose for TGF-beta, $5 \mathrm{ng} / \mathrm{ml}$, is used in several studies of MCF-7 cells (Mahdi et al. 2015; Noman et al. 2017; Tian and Schiemann 2017), and the medium dose we used for retinoic acid, $200 \mathrm{nM}$, is between the $100 \mathrm{nM}$ dose used in (Hua et al. 2009) and the 1 uM dose used in (Cunliffe et al. 2003). All conditions had the same $0.0125 \%$ concentration of ethanol. At 72 hours, we then trypsinized the cells in each well, removing 50,000 of them for immediate ATAC-seq library preparation and lysing the rest of them in Qiazol (storing immediately at $-80^{\circ} \mathrm{C}$ ) for subsequent RNA extraction and bulk RNA-seq library preparation.

\section{Immunofluorescence experiments and imaging}

For immunofluorescence experiments, we seeded 8-well glass chambers (Lab-tek 12-565-470) with hormone-starved MCF-7 cells for 24 hours before treating the cells with the medium dose of TGF-beta $(5 \mathrm{ng} / \mathrm{ml})$, retinoic acid $(200 \mathrm{nM})$, or vehicle $(0.0125 \%$ ethanol). Following treatment, we fixed cells for 12 minutes in 3.7\% formaldehyde (Sigma F1635) diluted in 1x PBS. We stored samples at 4C in 1x PBS, then performed the immunofluorescence protocol exactly as described by Cell Signaling Technology, using a dilution of 1:800 for the primary anti-pSMAD2 antibody (Cell Signaling Technology 18338T), 1:200 for the primary anti-RARA antibody (Sigma HPA058282), and 1:1000 for the goat anti-rabbit secondary antibody conjugated with Alexa Fluor 647 (Thermo Fisher Scientific A-21244). In brief, we blocked samples with $5 \%$ goat serum for 60 minutes, incubated with primary antibody overnight at $4 \mathrm{C}$, washed three times with 1 X PBS for 10 minutes each, incubated with secondary antibody at room temperature for 90 minutes in the dark, then washed the cells another three times in $1 \mathrm{X}$ PBS. We stained cellular nuclei with DAPI prior to imaging. We imaged the cells with an inverted Nikon TI-E microscope with a 20x Plan-Apo $\lambda$ (Nikon MRD00205) objective and with DAPI and Atto647N filter sets. We collected all images at 20x magnification.

\section{Immunofluorescence image analysis}

To quantify the nuclear pSMAD2 and RARA signal in our immunofluorescence experiments, we developed a custom image analysis pipeline in python that was centered around the usage of Cellpose (Stringer et al. 2020) to detect the nuclear boundaries of each cell. We first used the DAPI channel to manually select three to six high-quality images per condition. High quality images had minimal stacking of cells, little correlation between DAPI and immunofluorescence signal, and had well-focused nuclei throughout the image. We then used the DAPI channel images as input to Cellpose, with an expected diameter parameter of 32 pixels. Using Cellpose's identified nuclear boundaries, we then calculated the average intensity inside each nucleus using the corresponding immunofluorescence channel (pSMAD2 or RARA). To correct for differences in background, we then subtracted the average intensity of the annulus surrounding each nucleus in each image, using a disc-shaped structured element, the scipy binary_dilation function, and the nuclear mask matrix defined by Cellpose to generate the surrounding annulus for each nucleus. We then used this normalized nuclear intensity value for comparing the pSMAD2 and RARA levels between each condition. 
RNA extraction, library preparation and sequencing

We extracted RNA from previously frozen MCF-7 cell Qiazol lysates using the Qiagen miRNeasy kit (217004). We then used the NEBNext Ultra II RNA Library Prep Kit for Illumina (E7770) with the NEBNext Poly(A) mRNA Magnetic Isolation Module (E7490) and NEBNext Multiplex Oligos for Illumina (E7600) to prepare individual libraries. We then pooled our three replicates' libraries together and performed paired-end sequencing on an Illumina NextSeq 500, using a 75-cycle NextSeq 500/550 High Output Kit v2.5 (20024906), yielding 15 million read pairs per sample.

\section{RNA-sequencing analysis pipeline}

We aligned reads to the hg38 assembly using STAR v2.7.1a and counted uniquely mapped reads with HTSeq v0.6.1 and the hg38 GTF file from Ensembl (release 90). We performed differential expression analysis using DESeq2 v1.22.2 (Love et al., 2014) in R 3.5.1, using a minimum absolute-value log-fold-change of 0.5 and a q value of 0.05 . For genes with multiple possible transcription start sites, we used the genomic coordinates of the "canonical" transcription start site available in the knownCanonical table from GENCODE v29 in the UCSC Table Browser.

\section{ATAC library preparation and sequencing}

At the endpoint of each cell condition, we immediately performed the Omni-ATAC protocol (Corces et al., 2017) on 50,000 live MCF-7 cells, using Illumina Tagment DNA Enzyme TDE1 (20034197) at the tagmentation step and double-sided bead purification at the endpoint with Agencourt AMPure XP magnetic beads (A63880). The exact protocol we used is available in the protocols folder at https://github.com/emsanford/combined_responses_paper. We then performed paired-end sequencing using one 75-cycle NextSeq 500/550 High Output Kit v2.5 (20024906) for each replicate, yielding 42 million read pairs per sample.

\section{ATAC-sequencing analysis}

We created a paired-end read analysis pipeline using the ENCODE ATAC-seq v1 pipeline specifications (available at https://www.encodeproject.org/documents/c008d7bd-5d60-4a23-a833-67c5dfab006a/@@do wnload/attachment/ATACSeqPipeline.pdf). Briefly, we aligned our ATAC-seq reads to the hg38 assembly using bowtie2 v2.3.4.1, filtered out low-quality alignments with samtools v1.1, removed duplicate read pairs with picard 1.96, and generated artificial single-ended text-based alignment files containing inferred Tn5 insertion points with custom python scripts and bedtools v2.25.0. To call peaks, we used MACS2 2.1.1.20160309 with the command, "macs2 callpeak --nomodel --nolambda --keep-dup all --call-summits -B --SPMR --format BED -q 0.05 --shift 75 --extsize 150". While we created this pipeline for use on the Penn Medicine Academic Computing Services' high performance cluster, it is also publicly available at github.com/arjunrajlaboratory/atac-seq_pipeline_paired-end. Our pipeline generates a series of post-sequencing quality control metrics, which we have provided in Supplementary Table 1.

Since we had three biological replicates per ATAC-seq condition, we used an established "majority rule" to retain only the peak summits that were found in at least two 
replicates (Yang et al., 2014) (we used a peak size of $150 \mathrm{bp}$, centered on MACS2 summit locations, to mimic the span of one nucleosome). Using these condition-specific peak files, we then used bedtools to create one "master consensus peak file" by merging each condition's peak summit file together in a manner that disallowed overlapping peaks. We then used the number of ATAC-seq fragment counts at each peak in this master consensus peak file for differential peak analysis.

We wrote a custom peak analysis algorithm that took advantage of our additional ethanol control conditions to estimate a false discovery rate for differential peak identification. In this algorithm, we first count the number of ATAC-seq reads at each peak in the master consensus peak file. We then normalize the fragment counts at each peak to correct for differences in total sequencing depth. In this normalization step, we divide the number of reads in peaks for a given sample by $\frac{\text { sample's total number of reads in peaks }}{\text { average number of reads in peaks across all samples }}$. Then, for each condition, we calculate the average number of normalized read counts at each peak. Following this, we fill in an estimated false discovery rate in each cell of a 50x50 grid containing 50 exponentially-spaced steps of minimum fold-change values (ranging from 1.1 to 10) and 50 exponentially-spaced steps of minimum number of normalized fragment counts in the condition with the larger number of counts (ranging from 10 to 237). To calculate the estimated false discovery rate, we counted the number of differential peaks between signal-treated conditions and the normal density ethanol control as well as the number of differential peaks between additional ethanol controls (50\% and $150 \%$ starting cell density) and the normal density ethanol control. We then used the average number of differential peaks in the additional controls to estimate the number of false positive peaks per experimental condition, then calculated the final estimated false discovery rate (FDR) for a given parameter pair using the following formula:

$$
\text { estimated FDR }=\frac{\text { (number of conditions) }(\text { estimated number of false positive peaks per condition) }}{\text { total number of differential peaks in experimental conditions }}
$$

After calculating the estimated FDR for each cell of the $50 \times 50$ grid, we then pooled together the differential peaks contained in any cell containing an FDR less than $0.25 \%$. After pooling together the peaks in each of these cells and counting the number of differential peaks in the signal-treated conditions and additional controls, the combined estimated FDR was $0.65 \%$. We then noticed that our original peak set's fixed nucleosomal peak size of 150 bp led to many genomic regions containing several adjacent peaks that appeared to form a single, larger peak. Because of this, we merged our peaks together when they were within 250 base pairs of each other, then we performed a second round of the same differential peak calling algorithm on the merged peaks, requiring a minimum fold change of 1.5 and a minimum normalized fragment count value of 30 . In this final peak set, there are a total of 34,323 differential peaks, with a pooled estimated false discovery rate of $0.43 \%$.

We performed motif analysis on our set of differential peaks using chromVAR v1.5.0 (Schep et al., 2017), its associated curated cisBP database of transcription factor motifs, and the motifmatchR Bioconductor package. We treated each replicate as one sample for a given condition, and we pooled together the different dosages of the same signal(s) to decrease the variance of the transcription factor motif deviation scores for retinoic acid, TGF- $\beta$, and 
combined treatment. We slightly modified the chromVAR code to extract an internal metric that equals the fractional change in fragment counts at motif-containing peaks for a given motif.

\section{Statistical model for categorical classification of combined responses}

For a given gene in a given experimental condition, we assumed that its transcripts per million (TPM) value for one replicate was drawn from a Gaussian distribution. We estimated the parameters of these Gaussian distributions to create an $80 \%$ confidence interval for which to compare additive and multiplicative predictions. For each dosage of the combination treatment, we classified a gene as sub-additive if the additive and multiplicative predictions were higher than the $80 \%$ confidence interval, additive if only the additive prediction laid in the confidence interval, multiplicative if only the multiplicative prediction laid in the interval, super-multiplicative if both additive and multiplicative predictions were below the confidence interval, and ambiguous if both the additive and the multiplicative prediction laid within the interval.

To estimate the mean expression value of a gene in an experimental condition (e.g., 200 $\mathrm{nM}$ retinoic acid), we simply calculated the average TPM value across the three replicates. To improve our variance estimates, we took advantage of an observation we made during extensive manual review that the coefficient of variation $(\mathrm{CV})$ appeared to be the same between each dosage we tested for retinoic acid, TGF- $\beta$, and combined treatment (Supplemental Figure $7 \mathrm{E}-\mathrm{F})$. We then assumed that each dosage of a condition shared one CV term, which we calculated by averaging each dose's CV estimate using the unbiased estimator:

$$
\begin{gathered}
C V(\text { gene, signal, dosage })=\left(1+\frac{1}{4 n}\right)_{x}^{S} \\
C V(\text { gene, signal })=\frac{1}{m} \sum_{1}^{m} C V\left(\text { gene }, \text { signal }, \text { dosage }_{m}\right)
\end{gathered}
$$

Where $n$ is the number of replicates (3 in our case), $s$ is the sample standard deviation, and $\bar{x}$ is the mean of the measured TPM values, and $m$ is the number of doses tested ( 3 in our case). Finally, we used this averaged CV estimate to estimate a variance parameter for the Gaussian distribution we assumed to underlie the TPM values for a given gene and signal. For a given gene, dosage, and signal, our final estimated Gaussian distribution was:

TPM (gene, signal, dosage $) \sim$ Gaussian $\left(\overline{x_{\text {gene, signal, dosage }}},\left(\overline{x_{\text {gene, signal, dosage }}} \times C V(\text { gene, signal })\right)^{2}\right)$ Where $\overline{x_{\text {gene, signal, dosage }}}$ is the measured average TPM value for a given gene exposed to a specific dose of retinoic acid, TGF- $\beta$, or combination treatment. The benefit of using our shared $\mathrm{CV}$ term across dosages was to move from using the information from 3 samples to using the information from 9 samples when estimating the variances of these distributions.

To classify ATAC-seq peaks as sub-additive, additive, or super-additive, we used the same approach described above for RNA-seq TPM values, but with a given peak's normalized fragment count value. We then classified peaks as sub-additive or super-additive if the additive prediction was higher than or lower than (respectively) the estimated Gaussian distribution's $80 \%$ confidence interval.

Statistical model for simulated additive and multiplicative predictions 
To simulate new ATAC-seq and RNA-seq measurements, for each gene and condition we randomly sampled three new observations from a folded Gaussian distribution (folded to avoid negative expression or normalized fragment count values) with the parameters we previously estimated for the purpose of categorically classifying combined response behaviors. For the combined treatment, we set the mean of the distribution to be either a perfectly additive or perfectly multiplicative prediction. We then calculated the average of the three new simulated observations and used these average values to determine a gene's $c$ value at a given dose or an ATAC-seq peak's d-value at a given dose. Using this process, we calculated 250 simulated $c$ values for each dose of each upregulated gene in our master set and 10 simulated $d$ values for each ATAC-seq peak that was upregulated individually by retinoic acid and TGF- $\beta$. In the simulated data mixture model where genes can be strictly additive or multiplicative, at each we randomly assigned a gene to be additive or multiplicative based on the ratio of the dose-specific frequencies we observed in the categorical classification of the combined response.

Use of simulated data to infer the location of a secondary peak in the observed combined response factor (c value) histogram

To generate a hypothetical plot of observed $c$ values in which the primary peak of additive responses centered at $\mathrm{c}=0$ was depleted, we subtracted the additive component of a $c$ value histogram generated by simulated data. These simulated $c$ values were generated using gene and condition-specific Gaussian distributions in a process outlined above and in Supplemental Figure 7. At each dose, we simulated data as a mixture of additive and multiplicative combined responses, setting the exact proportion of simulated additive versus multiplicative combined responses based on the ratio of additive to multiplicative combined transcriptional responses seen at each dose of the observed data (Figure 1E; Supplemental Figure 7B). We then scaled the size of this "mixed" simulated c value distribution to the peak heights at $c=0$ and $c=1$ in the observed $c$ value histogram by minimizing the squared distance between the simulated and observed histogram bars directly abutting $c=0$ and $c=1$ (4 histogram bars total). We then subtracted the additive component of the simulated $c$ value distribution and locally (in the range of $c=-4$ to $c=5$ ) fit a Gaussian density function to the residual histogram using the nls function in $\mathrm{R}$.

We also estimated the probability of obtaining the number of combined transcriptional responses in each bin of our observed $c$ value histogram if all combined responses were additive. To do this, we scaled the peak height of our observed data at $c=0$ to the peak height of an additively simulated distribution of $c$ values. We then repeatedly (1,000 times) ran new simulations of additive combined responses, simulating one observation per gene in our master set of 1,384 genes. We used a bin width of 0.25 and allowed for overlapping bins. Because the probability of obtaining the observed number of counts was extremely low for many bins and because the variability in the number of observations in a given bin was well described by a Poisson distribution (outside the range of $-0.3<c<0.3$ ), we used a Poisson cumulative density function to estimate the probability of witnessing the number of observed counts (or greater) in each c value bin of the simulated additive data. 
Generating a null distribution for dual-motif matches

To generate a null distribution for dual-motif matches, we first separated our set of upregulated peaks into sub-additive, additive, and super-additive peaks. Within these peak subsets, we counted the number of retinoic acid-dominant (FOX, and ETS-family factors), TGF- $\beta$-dominant (SMAD, AP-1, BACH, BATF, SMARCC1, NFE2, NFE2L2, MAFF, and MAFK), and neither-signal-dominant (HOX, NFKB, CDX, CTCF, BCL, and GRHL1) motifs at each peak. Due to similar features of their position-weight matrices, we avoided over-counting similar motifs by reporting the maximum number of motif matches for a single type of motif within a group of motifs. The motif groups we used were as follows: retinoic acid receptor consisted of RARA, group FOX consisted of FOXA1, FOXA2, FOXA3, FOXC2, FOXD3; group ETS consisted of SPI, SPIB, SPIC, EHF, ELF1, ELF2, ELF3, ELF4, ELF5; group SMAD consisted of SMAD3, SMAD4, SMAD9; group AP-1 consisted of JUN, JUNB, JUND, JDP2, FOS, FOSB, FOSL1, FOSL2, BACH1, BACH2, BATF (note the inclusion of non-canonical AP-1 factors due to their similar motif position weight matrices); group SMARCC1 consisted of SMARCC1; group NFE consisted of NFE2, NFE2L2; group MAF consisted of MAFF, MAFK; group HOX consisted of HOXA13, HOXB13, HOXC10, HOXC12, HOXC13, HOXD13; group NFKB consisted of NFKB1, REL, RELA; group CDX consisted of CDX1, CDX2; group CTCF consisted of CTCF; group BCL consisted of BCL11A, BCL11B; group GRHL1 consisted of GRHL1. For example, if a peak had three JUN motifs, two FOS motifs, two JDP2 motifs, and one BACH1 motif, we would count this as three AP-1 motifs. We then randomly shuffled these grouped motif matches within each peak set, with each peak retaining its original number of total motif matches (thus a peak with zero motif matches also had zero motif matches and a peak with four grouped motif matches always had four grouped motif matches after each random shuffle). After each of 1,000 random shuffles, we calculated the fraction of peaks in each peak set that contained both a retinoic acid-dominant and a TGF- $\beta$ dominant motif.

\section{Statistical analysis}

With the exception of DESeq2's adjusted $p$ value and our manually calculated $p$ value for the null distribution we generated for dual-motif matches at upregulated ATAC-seq peaks, we calculated all reported $p$ values in the figures and main text using Welch's unequal variances t-test in R. (Note that we did not correct for multiple comparisons.)

\section{Data and code availability}

All custom data analysis code is available at https://github.com/emsanford/combined responses paper

Raw ATAC-seq and RNA-seq data is available at https://www.dropbox.com/sh/fhx7huyhhtf8fux/AACKW5Bd7k34uy6Rrk3k0WZ4a?dl=0\&lst=

The ATAC-seq pipeline we used is available at https://github.com/arjunrajlaboratory/atac-seq pipeline paired-end

The RNA-seq pipeline we used is available at https://github.com/arjunrajlaboratory/RajLabSeqTools 


\section{Box 1: mathematical description of additive versus multiplicative combined transcriptional responses}

Suppose that gene $X$ is expressed at baseline and increases its transcription in response to either signal A or signal B:

$$
\begin{aligned}
& \text { expression of gene } X \text { at baseline }=X_{\text {baseline }} \\
& \text { expression of gene } X \text { after receiving signal } \mathrm{A}=X_{\text {baseline }}+\Delta_{A} \\
& \text { expression of gene } X \text { after receiving signal } \mathrm{B}=X_{\text {baseline }}+\Delta_{B}
\end{aligned}
$$

If the combined transcriptional response to receiving both signals $\mathrm{A}$ and $\mathrm{B}$ were additive, the increase in transcription of gene $\mathrm{X}$ would reflect the sum of the effects $\Delta_{A}$ and $\Delta_{B}$ :

$$
\text { additive combined response of gene } X \text { to signals } \mathrm{A} \text { and } \mathrm{B}=X_{\text {baseline }}+\Delta_{A}+\Delta_{B}
$$

If the combined response were multiplicative, the increase in transcription of gene $X$ would reflect the product of the fold change experienced under signals A and B:

$$
\begin{aligned}
\text { multiplicative combined response } & =X_{\text {baseline }} \times \text { fold-change }_{A} \times \text { fold-change }_{B} \\
& =X_{\text {baseline }} \times \frac{X_{\text {baseline }}+\Delta_{A}}{X_{\text {baseline }}} \times \frac{X_{\text {baseline }}+\Delta_{B}}{X_{\text {baseline }}}
\end{aligned}
$$

Multiplying out the terms of the previous expression, we see that the difference between an multiplicative and additive combined response is exactly $\frac{\Delta_{A} \times \Delta_{B}}{X_{\text {baseline }}}$ :

$$
\begin{aligned}
\text { multiplicative response } & =X_{\text {baseline }} \times \frac{X_{\text {baseline }}+\Delta_{A}}{X_{\text {baseline }}} \times \frac{X_{\text {baseline }}+\Delta_{B}}{X_{\text {baseline }}} \\
& =X_{\text {baseline }} \times \frac{X_{\text {baseline }}^{2}+\left(X_{\text {baseline }} \times \Delta_{A}\right)+\left(X_{\text {baseline }} \times \Delta_{B}\right)+\left(\Delta_{A} \times \Delta_{B}\right)}{X_{\text {baseline }}^{2}} \\
& =\frac{X_{\text {baseline }}^{2}+\left(X_{\text {baseline }} \times \Delta_{A}\right)+\left(X_{\text {baseline }} \times \Delta_{B}\right)+\left(\Delta_{A} \times \Delta_{B}\right)}{X_{\text {baseline }}} \\
& =X_{\text {baseline }}+\Delta_{A}+\Delta_{B}+\frac{\Delta_{A} \times \Delta_{B}}{X_{\text {baseline }}} \\
& =\text { additive response }+\frac{\Delta_{A} \times \Delta_{B}}{X_{\text {baseline }}}
\end{aligned}
$$

We defined a term, $c$, the combined response factor, that can be determined after measuring a gene's expression at baseline and in response to both single and combined signal treatments:

$$
\text { gene } X \text { 's combined response }=X_{\text {baseline }}+\Delta_{A}+\Delta_{B}+c \times \frac{\Delta_{A} \times \Delta_{B}}{X_{\text {baseline }}}
$$

For a gene that increases transcription in response to both signals, the combined response is perfectly additive when $c=0$, perfectly multiplicative when $c=1$, sub-additive when $c<0$, and super-multiplicative when $c>1$. Thus, a gene's combined response factor, which can be solved for after profiling gene expression in unperturbed and signal-treated cells, provides us with a metric for describing combined transcriptional responses along a continuum that spans addition and multiplication. 
bioRxiv preprint doi: https://doi.org/10.1101/2020.05.26.116962; this version posted October 27, 2020. The copyright holder for this preprint (which was not certified by peer review) is the author/funder, who has granted bioRxiv a license to display the preprint in perpetuity. It is made available under aCC-BY 4.0 International license.

Box 1: Mathematical formulation of an additive combined response, multiplicative combined response, and the combined response factor (c value) 
bioRxiv preprint doi: https://doi.org/10.1101/2020.05.26.116962; this version posted October 27, 2020. The copyright holder for this preprint (which was not certified by peer review) is the author/funder, who has granted bioRxiv a license to display the preprint in perpetuity. It is made available under aCC-BY 4.0 International license.

\section{Figure 1}

A Suppose Gene $X$ receives two signals:

$$
\begin{aligned}
& \begin{aligned}
100+\text { signal } A & \\
\text { \# mRNA copies/cell } & 200 \\
\Delta_{A} & =100 \\
\text { fold-change } & =2
\end{aligned} \\
& 100 \stackrel{+ \text { signal } B \longrightarrow}{\longrightarrow} 300
\end{aligned}
$$

if the signal effects add:

$$
100 \stackrel{+ \text { signal } A \text { and signal } B}{\left(100+\Delta_{A}+\Delta_{B}\right)}
$$

if the signal effects multiply:

$$
100 \stackrel{+ \text { signal } A \text { and signal } B}{\underset{\left(100 \times \text { fold-change }_{A} \times \text { fold-change }_{B}\right)}{\longrightarrow}} 600
$$

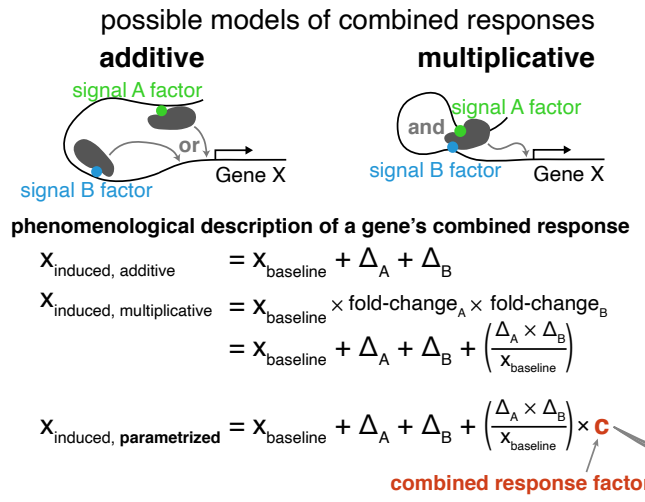

possible models of combined responses

Gene X, possible integration outcomes
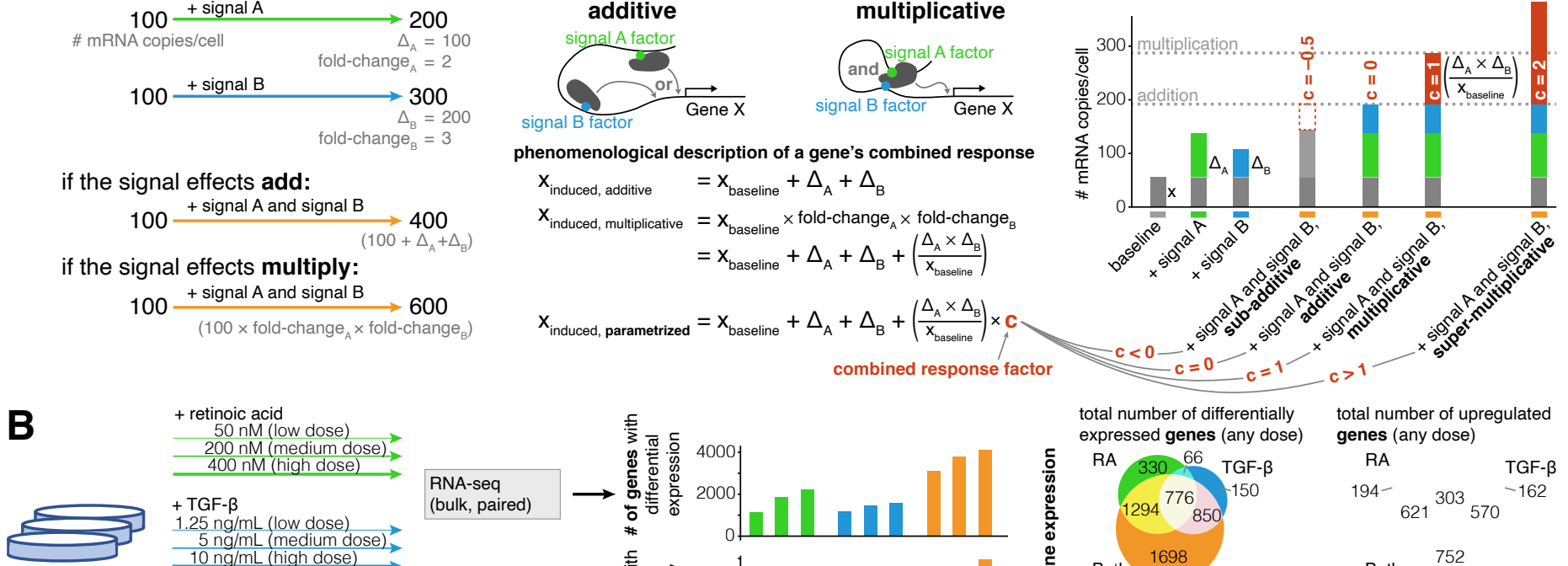

+ retinoic acid
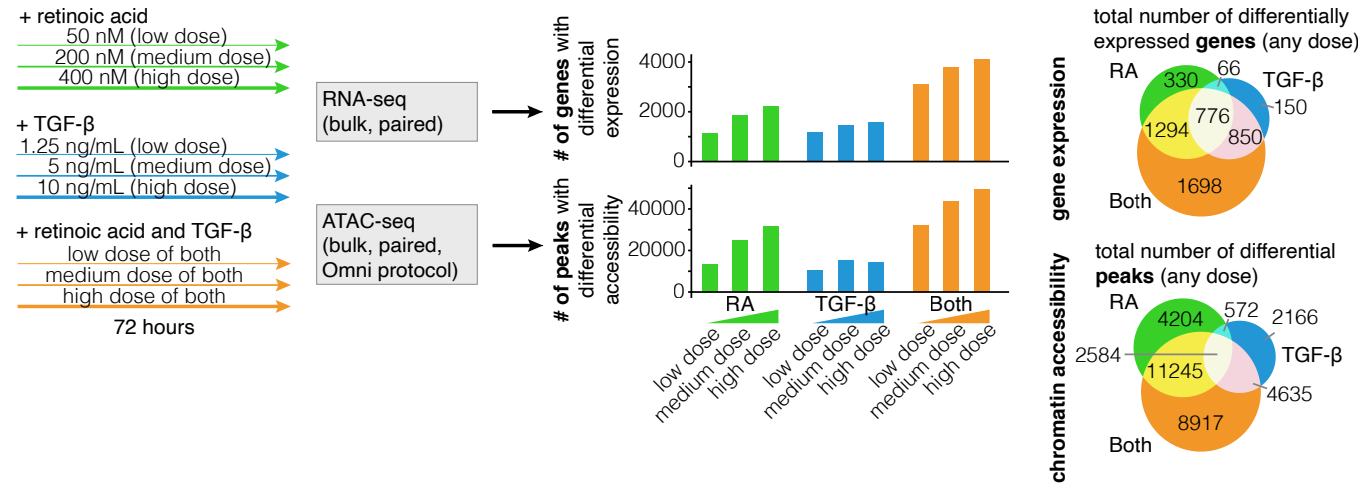

total number of upregulated genes (any dose)

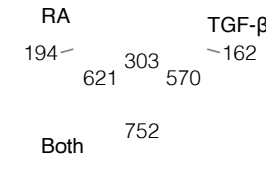

total number of upregulated peaks (any dose)

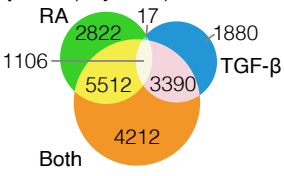

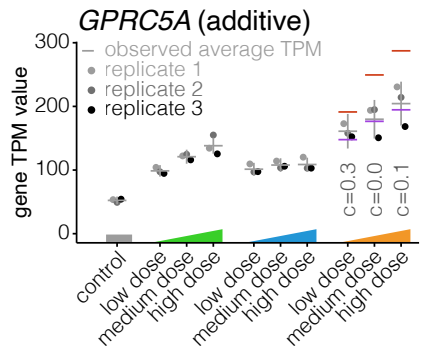

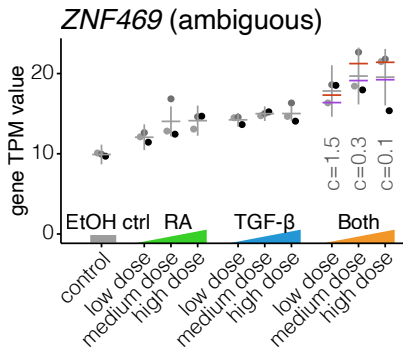

$\mathbf{F}$

observed $\mathbf{c}$ value distribution (from $N=1,384$ genes $^{*}$ )
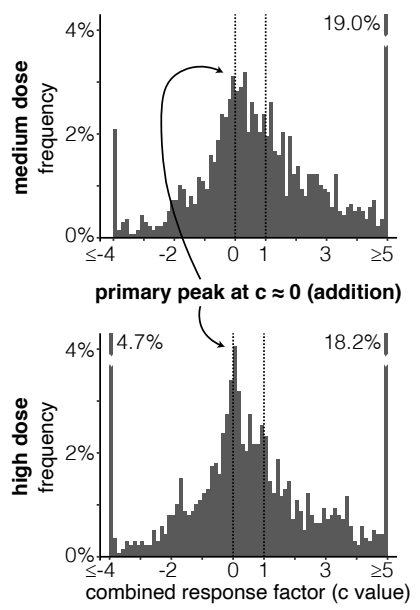

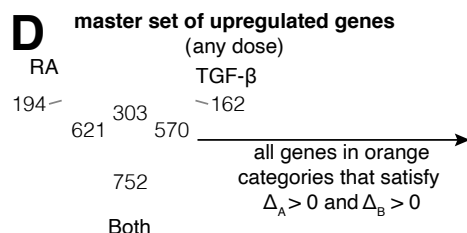

(total in all overlapping orange categories $=2,246$ )

simulated data: expecte additive
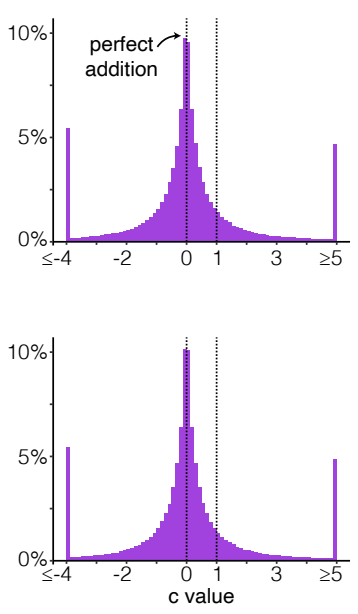

EPHB2 (multiplicative)
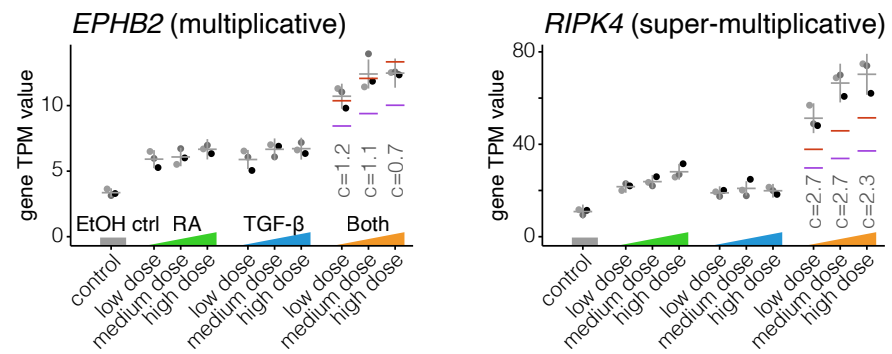

E classification of master set of genes upregulated by retinoic acid and TGF- $\beta$ ( $N=1,398)$

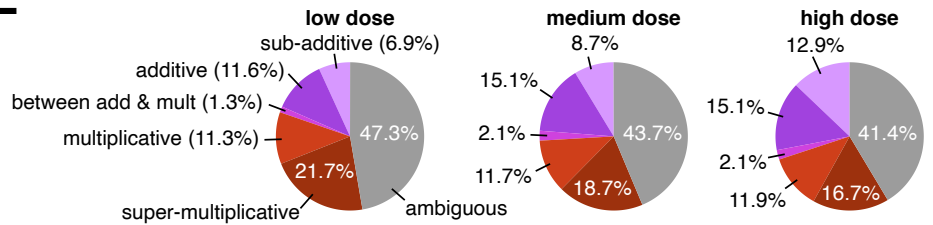

sured $c$ values if all combined responses were...

multiplicative
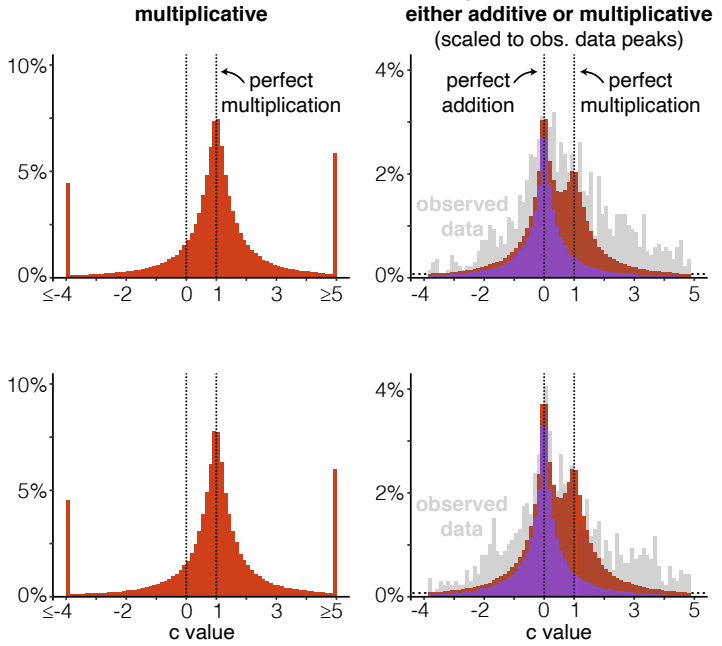

residual distribution after subtracting additive component

of mixed simulated data

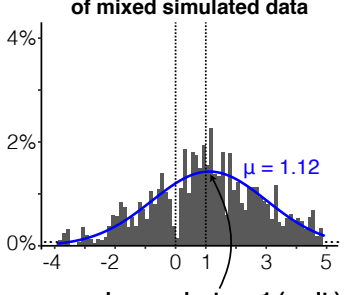

secondary peak at $c \approx 1$ (mult.)

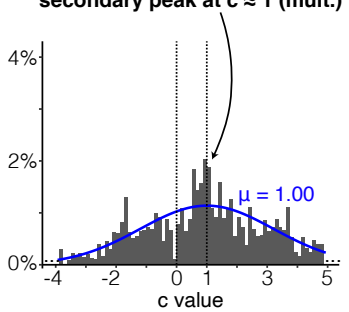


Figure 1: Addition and multiplication are enriched modes of signal integration in upregulated genes. A. Example of additive vs. multiplicative effects on expression of hypothetical gene $X$, mathematical formulation of the combined response factor, and illustration of how the value of the combined response factor (c value) reflects whether a combined gene expression response is sub-additive, additive, multiplicative, or super-multiplicative. B. Schematic of signal response experiments in MCF-7 cells. Briefly, we treated MCF-7 cells with three different dosages of retinoic acid, TGF- $\beta$, or both signals for 72 hours, then performed bulk RNA-seq and ATAC-seq at the endpoint. We show the number of differentially expressed genes and peaks for each dose of each condition as well as the overlap between the sets of differentially expressed genes and differential peaks. C. Five example genes representing sub-additive to super-multiplicative combined transcriptional responses, where we show each gene's transcripts per million (TPM) value for each replicate after single or combined signal treatments. Horizontal grey bars show the average TPM value, and error bars represent the $80 \%$ confidence interval of the estimated underlying Gaussian distribution of each dosage and condition (see Methods for parameter estimation details). D. Illustrated definition of master set of upregulated genes. E. Frequency of each type of combined response behavior for each dosage in the master set of genes. F. Simulated, observed, and residual histograms of $\mathrm{c}$ value distributions for the medium and high doses. In the simulated mixture model, we randomly simulated combined responses to be either additive or multiplicative based on the relative frequency of additive vs. multiplicative combined transcriptional responses that we observed at each dose in 1E. Annotated percentages at broken bars represent the fraction of $c$ values in the tail beyond the limits of the $x$ axis of the graph. *for all $c$ value analyses, 14 genes with a control TPM of zero were removed from the master set of genes, as they end up misleadingly having $c$ values of exactly 0 regardless of the effects of retinoic acid and TGF-beta. 
bioRxiv preprint doi: https://doi.org/10.1101/2020.05.26.116962; this version posted October 27, 2020. The copyright holder for this preprint (which was not certified by peer review) is the author/funder, who has granted bioRxiv a license to display the preprint in perpetuity. It is made available under aCC-BY 4.0 International license.

\section{Figure 2}

A

EtOH control

RA $\quad \begin{aligned} & \text { low dose } \\ & \text { hed dose } \\ & \text { high dose }\end{aligned}$
TGF- $\beta$ med dose
high dose
Both
low dose
med dose
high dose

differential peaks

peak d value (med dose)

$$
\text { RARA }
$$

motif matches SMAD3

SMAD4
SMAD9

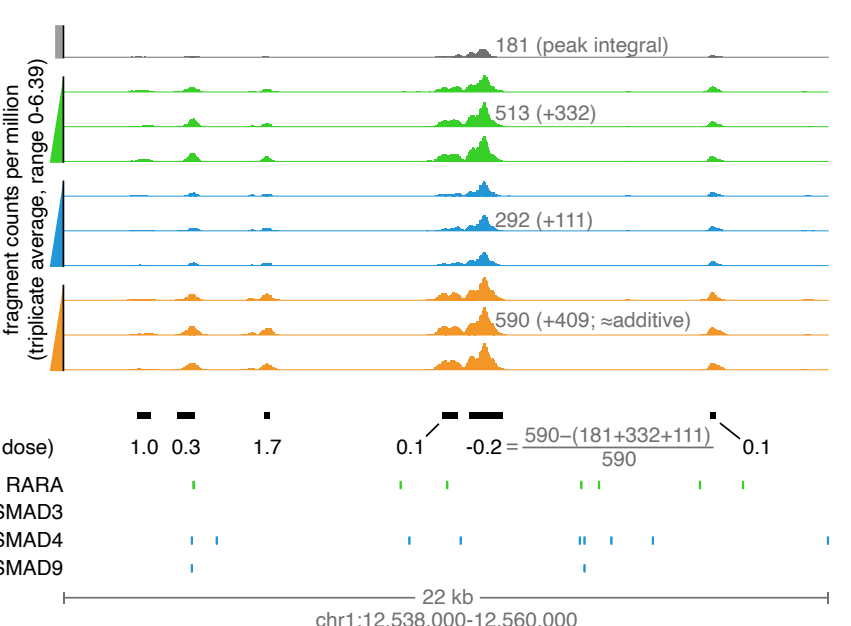

chr1:12,538,000-12,560,000

\section{ATAC-seq tracks}

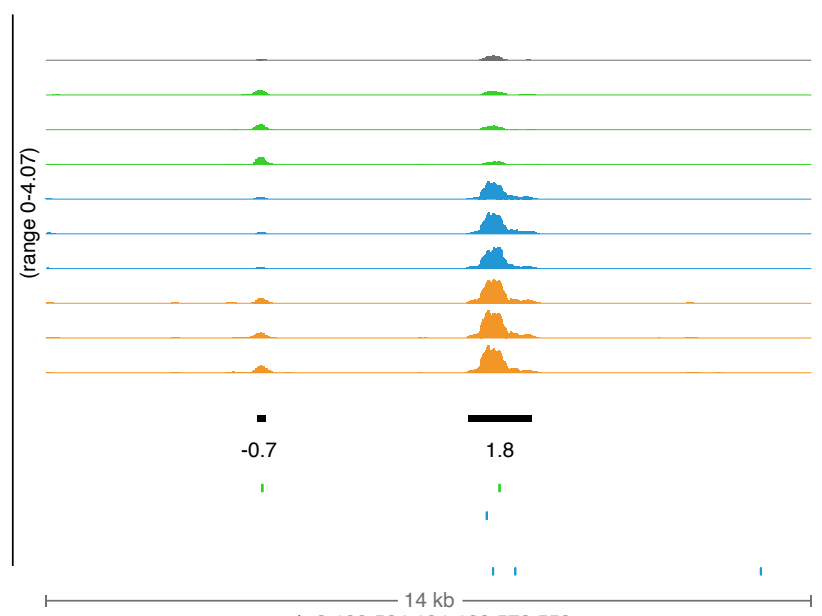

chr3:189,564,184-189,578,559

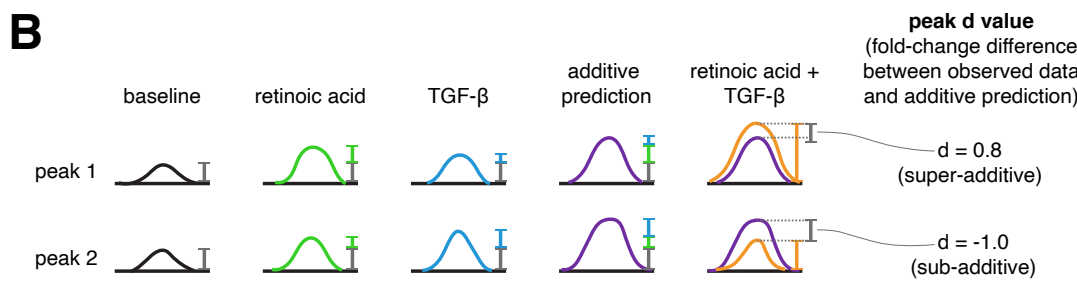

C

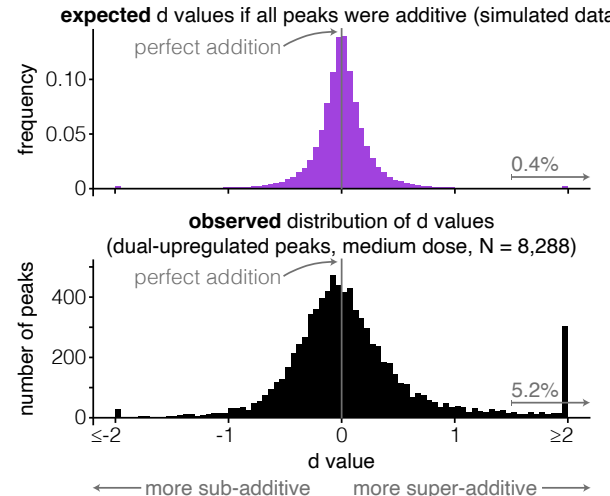

D

classification of peaks upregulated individually by retinoic acid and TGF- $\beta$ $(\mathrm{N}=8,288$, medium dose $)$

sub-additive

super-additive $16 \%$

$65 \%$

E addition is the observed type of combined response at peaks

Ehen increases in chromatin accessibility are independent and rare

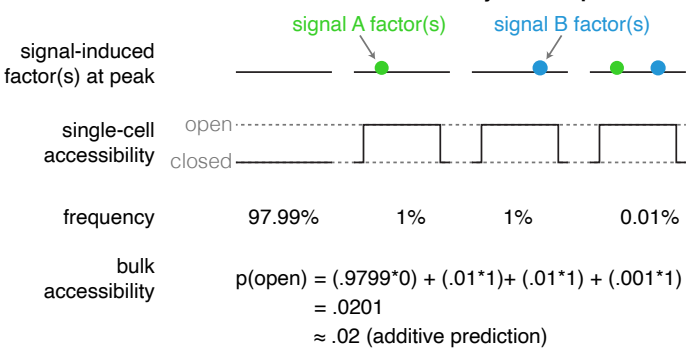


Figure 2: Addition is the default operation at upregulated differential peaks. A. Example tracks of ATAC-seq data. Tracks illustrate the ATAC-seq fragment counts per million, with each value representing the average number of fragment ends per million within $75 \mathrm{bp}$ of a given genomic coordinate. Annotated peak values represent the peak integral (the total number of normalized fragment counts measured within the peak), which we use to calculate the peak's d value. B. Schematic illustrating examples of two peak's d values, where each d value represents the fold-change difference between the measured number of ATAC-seq counts in the combination treatment and the predicted number of ATAC-seq fragment counts when using an additive model. C. Expected vs. observed distributions of the fold-change difference from an additive prediction for each peak. We generated the expected distribution by simulating 10 new observations for each peak from the distributions we estimated our original upregulated peaks to have come from, setting the mean of the combined treatment to a perfectly additive prediction (Methods). D. Classification of ATAC-seq peaks that were upregulated individually by retinoic acid and TGF- $\beta$. We considered a given peak to be additive when the additive model prediction lied within the $80 \%$ confidence interval of our estimated distribution of the given peak's normalized fragment counts in the combined treatment condition. E. Schematic illustrating how combined binding responses may be additive when transcription factor binding is independent and rare. 
bioRxiv preprint doi: https://doi.org/10.1101/2020.05.26.116962; this version posted October 27, 2020. The copyright holder for this preprint (which was not certified by peer review) is the author/funder, who has granted bioRxiv a license to display the preprint in perpetuity. It is made available under aCC-BY 4.0 International license.

\section{Figure 3}

A gene Y: additive combined transcriptional response

gene Z: multiplicative combined transcriptional response

sub-additive peak

1 super-additive, 2 additive ATAC-seq peaks nearby
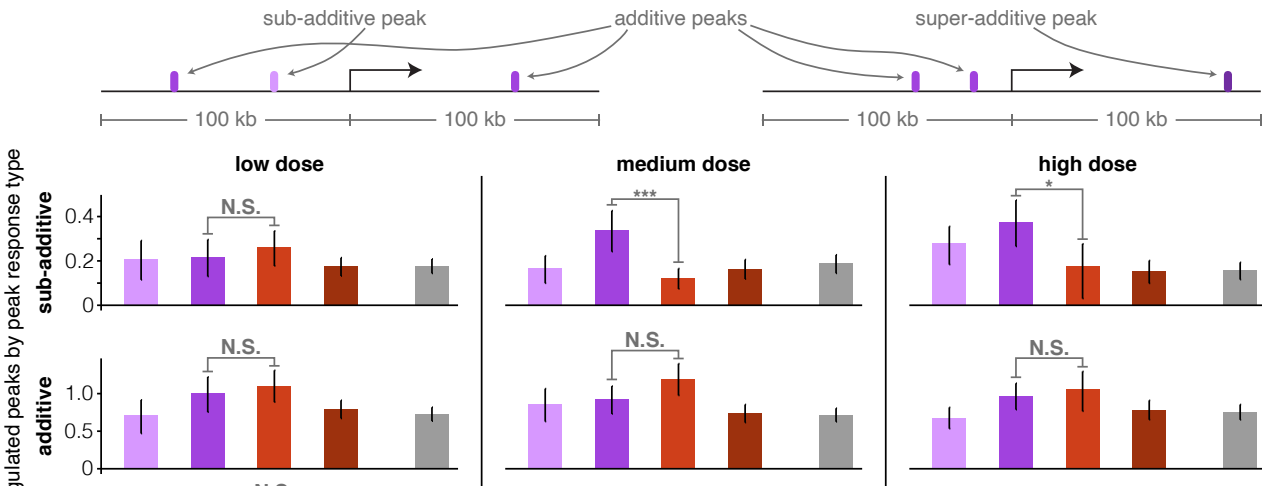

告
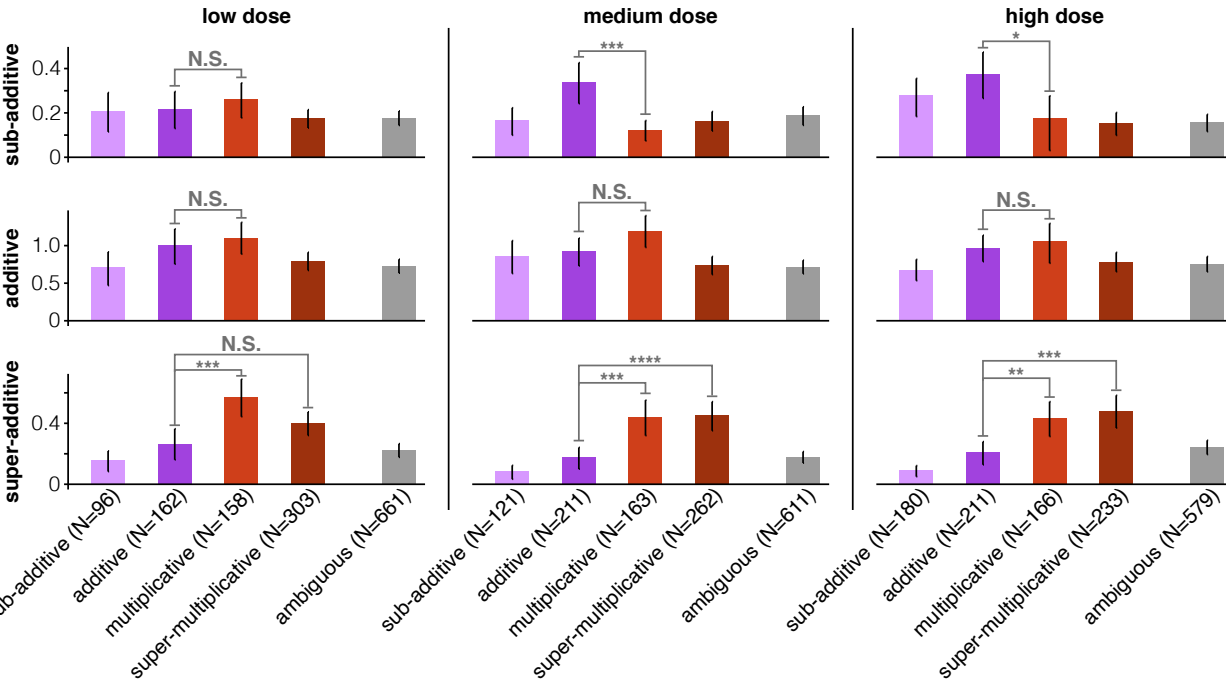

type of combined transcriptional response

(grouped by combined response classification shown in fig 1E)

B

gene A: multiplicative combined transcriptional response

has an RA and a TGF- $\beta$-dominant peak nearby

gene $B$ : additive combined transcriptional response peak opens only in response to RA peaks open only in response to TGF- $\beta$ heaks and a
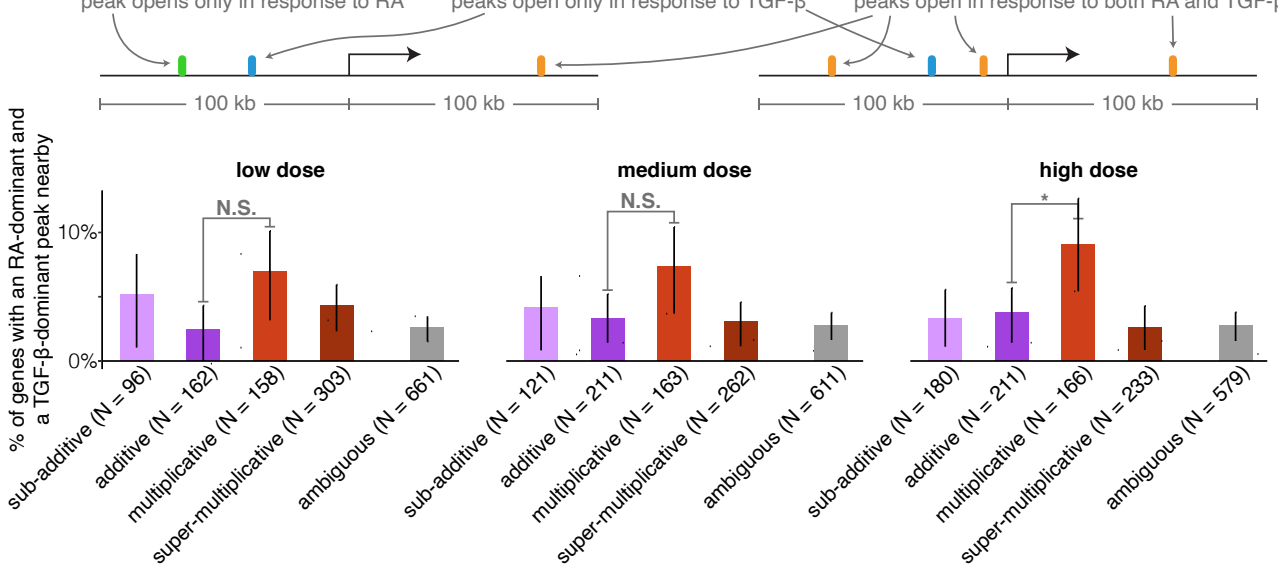

type of combined transcriptional response

(grouped by combined response classification shown in fig $1 \mathrm{E}$ ) 
Figure 3: Super-additive ATAC-seq peaks are enriched near genes with multiplicative and super-multiplicative combined transcriptional responses. A. For each type of combined gene expression response, we show the average number of upregulated sub-additive, additive, and super-additive ATAC-seq peaks within $100 \mathrm{~kb}$ of the gene's transcription start site. B. For each combined transcriptional response behavior, we show the percentage of genes that have at least one peak that responds exclusively to retinoic acid and at least one peak that responds exclusively to TGF- $\beta$ (where both peaks must lie within $100 \mathrm{~kb}$ of the gene's transcription start site). For an upregulated peak to be considered a mutually exclusive response, the change in ATAC-seq fragment counts in the individual treatment condition must be at least $9 x$ larger in the major signal effect than the minor signal effect. ${ }^{*} p<0.05 ;{ }^{* *} p<0.01$; ${ }^{* \star *} p<0.002$; ${ }^{* \star *} p<$ 0.0002. All $p$ values were calculated using Student's t-test. All error bars represent the $90 \%$ confidence interval estimated using 10,000 empirical bootstrap samples of the peak sets used in each analysis. 
bioRxiv preprint doi: https://doi.org/10.1101/2020.05.26.116962; this version posted October 27, 2020. The copyright holder for this preprint (which was not certified by peer review) is the author/funder, who has granted bioRxiv a license to display the preprint in perpetuity. It is made available under aCC-BY 4.0 International license.

\section{Figure 4}

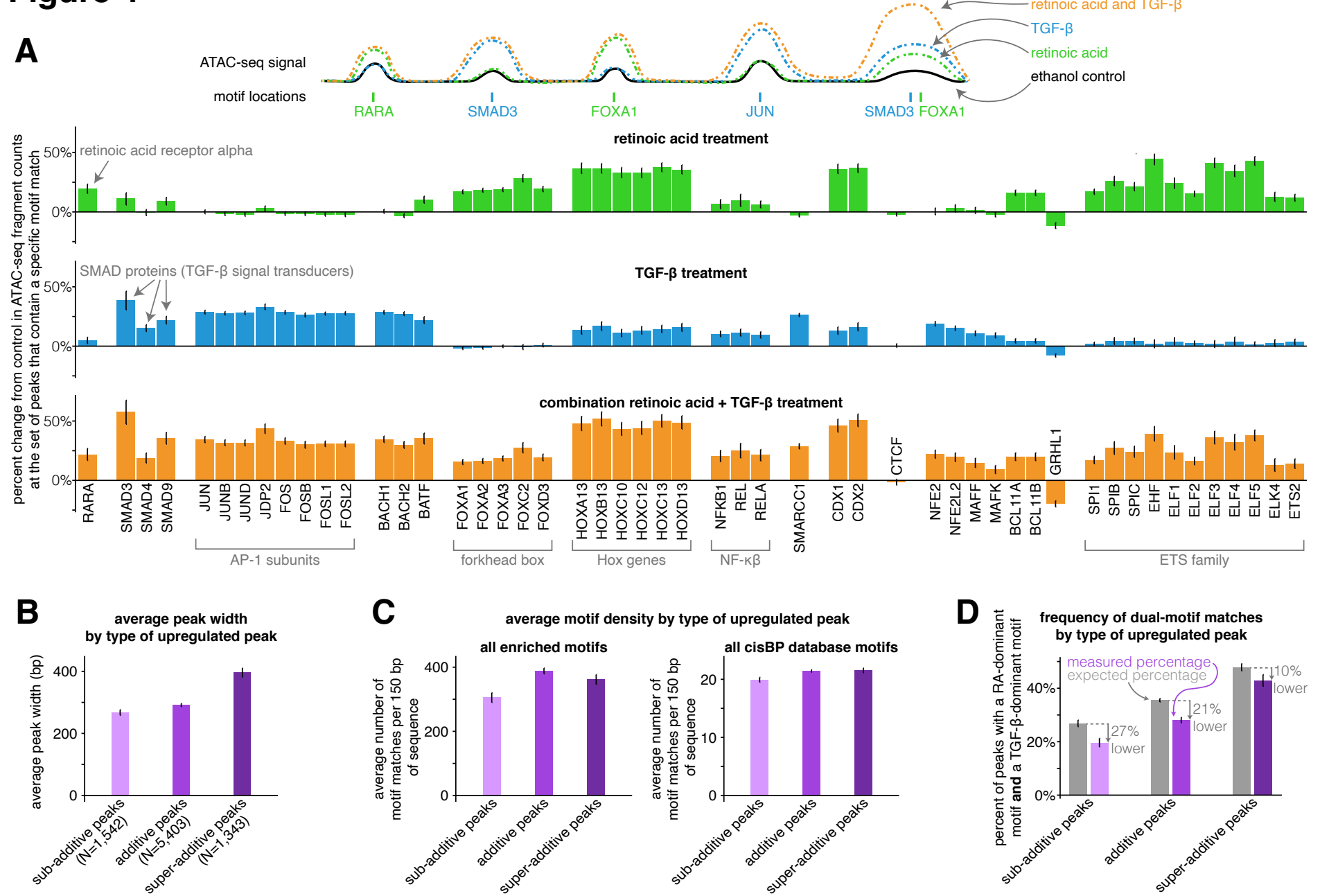

E

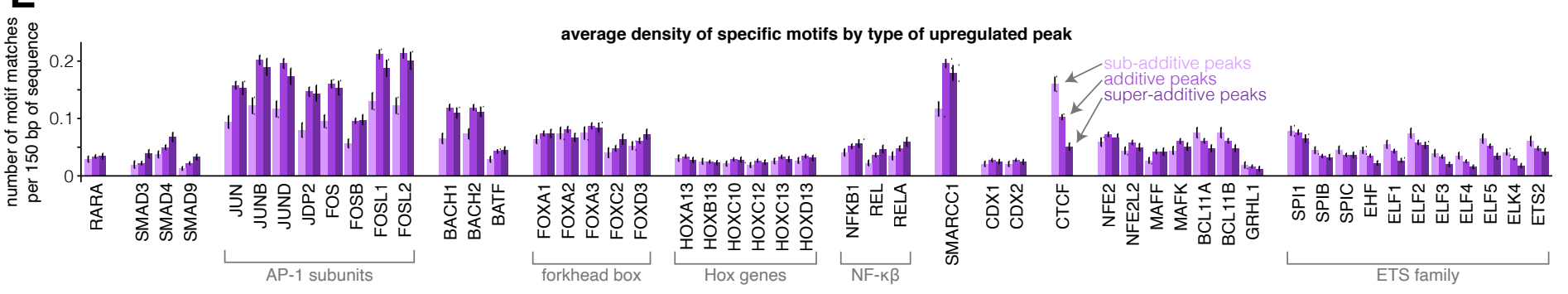

$\mathbf{F}$
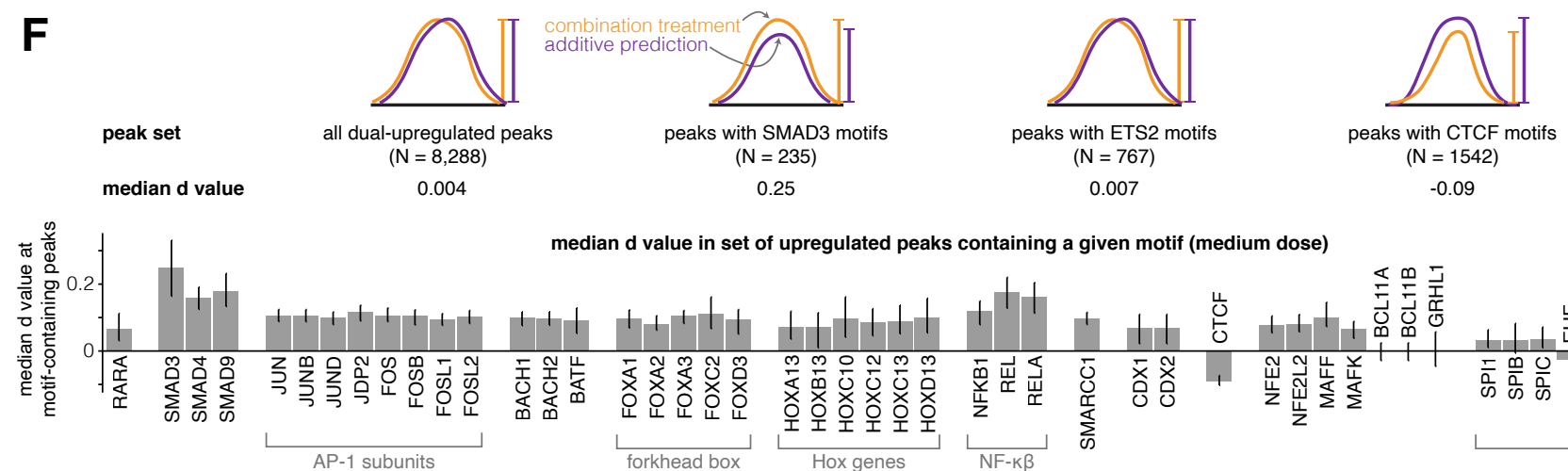

median $\mathbf{d}$ value in set of upregulated peaks containing a given motif (medium dose)

AP-1 subunits

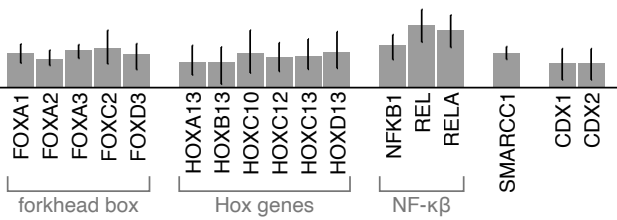

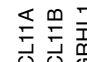


Figure 4: Sub-additive peaks are depleted for AP-1 motifs, enriched for CTCF motifs, while super-additive peaks are enriched for SMAD motifs. A. Motif enrichment analysis in each condition for the top 50 most variable transcription factor motifs identified by chromVAR. (CTCF was manually added to this set, making the total 51). Y-axis represents the percentage change in ATAC-seq signal at motif-containing peaks compared to ethanol control samples. For each condition, we pooled together the replicates for each of the three dosages, resulting in nine replicates each for retinoic acid, TGF- $\beta$, and combination treatment. B. Average peak width of peaks upregulated individually by retinoic acid and TGF- $\beta$ by type of combined response. C. Average motif density in each type of peak upregulated individually by retinoic acid and TGF- $\beta$, using the enriched motif set and the full cisBP database. D. Expected vs. measured percentage of dual-motif matches (one retinoic acid-dominant motif and one TGF- $\beta$ dominant motif) for each type of upregulated peak. We calculated the expected percentage by randomly shuffling motif matches within each peak set (see methods for details). Error bars represent the 5th and 95th percentile of the null distribution for expected percentages and the $90 \%$ bootstrapped confidence interval for measured percentages. E. Motif density by type of upregulated peak for each motif in our enriched set. F. For a given enriched motif, the median $\mathrm{d}$ value at medium dose for all upregulated peaks that contain the motif (higher $\mathrm{d}$ values indicate more super-additivity in peaks containing a given motif; the median d value for all upregulated peaks was 0.004). All error bars (except for the error bars for expected percentages in D) represent the $90 \%$ confidence interval estimated using 1,000 empirical bootstrap samples of the peak sets used in each analysis. 
bioRxiv preprint doi: https://doi.org/10.1101/2020.05.26.116962; this version posted October 27, 2020. The copyright holder for this preprint (which was not certified by peer review) is the author/funder, who has granted bioRxiv a license to display the preprint in perpetuity. It is made available under aCC-BY 4.0 International license.

\section{Supplemental Figure 1}

A

$$
\begin{aligned}
& \mathrm{x}_{\text {combination treatment }}=\mathrm{x}_{\text {baseline }}+\Delta_{\mathrm{A}}+\Delta_{\mathrm{B}}+\frac{\Delta_{\mathrm{A}} \times \Delta_{\mathrm{B}}}{\mathrm{x}_{\text {baseline }}} \times \mathrm{c} \\
& \text { combined response factor }(\mathrm{c})=\frac{\mathrm{x}_{\text {baseline }}\left(\mathrm{x}_{\text {combination treatment }}-\left(\mathrm{x}_{\text {baseline }}+\Delta_{\mathrm{A}}+\Delta_{\mathrm{B}}\right)\right)}{\Delta_{\mathrm{A}} \times \Delta_{\mathrm{B}}}
\end{aligned}
$$

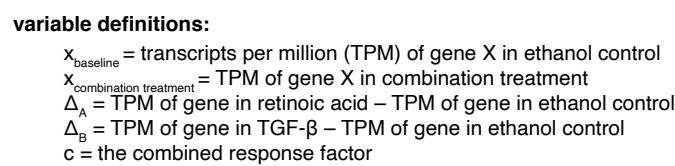

B change in each upregulated gene's $\mathrm{c}$ value following increases in signal dose

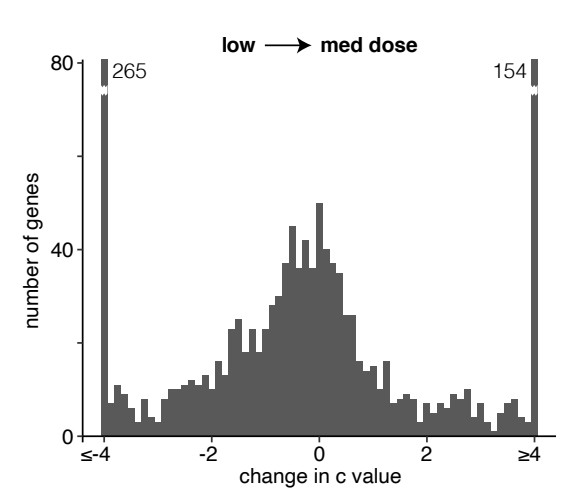
$(\mathrm{N}=1,384$ at each dose $)$
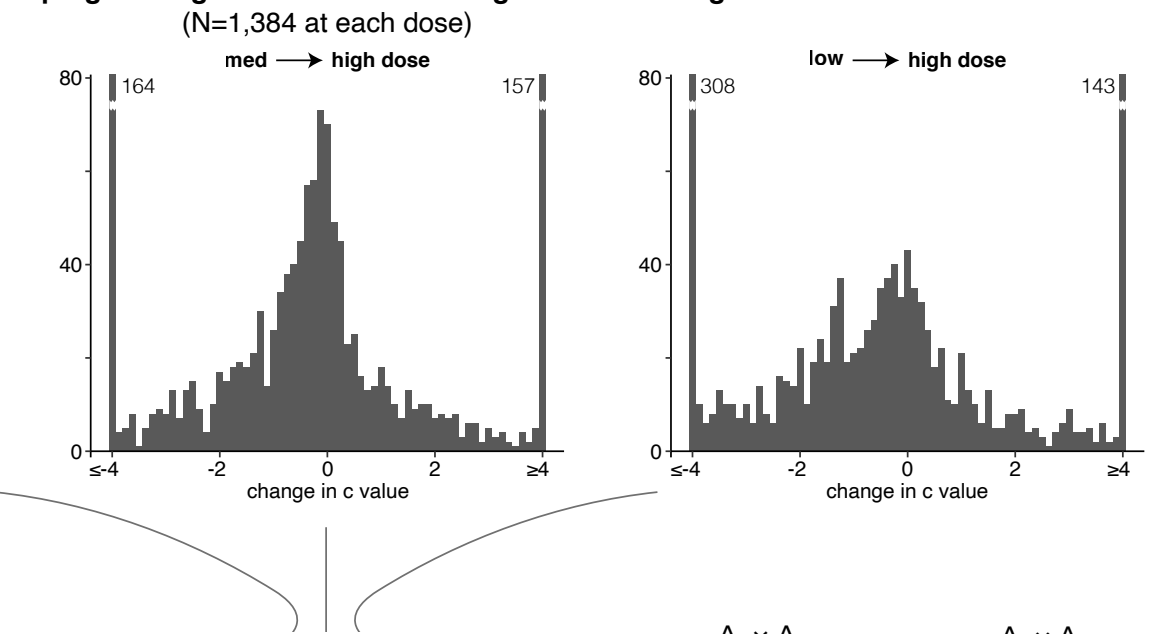

C subset of highly reliable $\mathrm{c}$ value estimates: require the combined responses at each dose to satisfy $\frac{\Delta_{A} \times \Delta_{B}}{X_{\text {baseline }}} \geq x_{\text {baseline }}$ and $\frac{\Delta_{A} \times \Delta_{B}}{X_{\text {baseline }}} \geq 2$ TPM
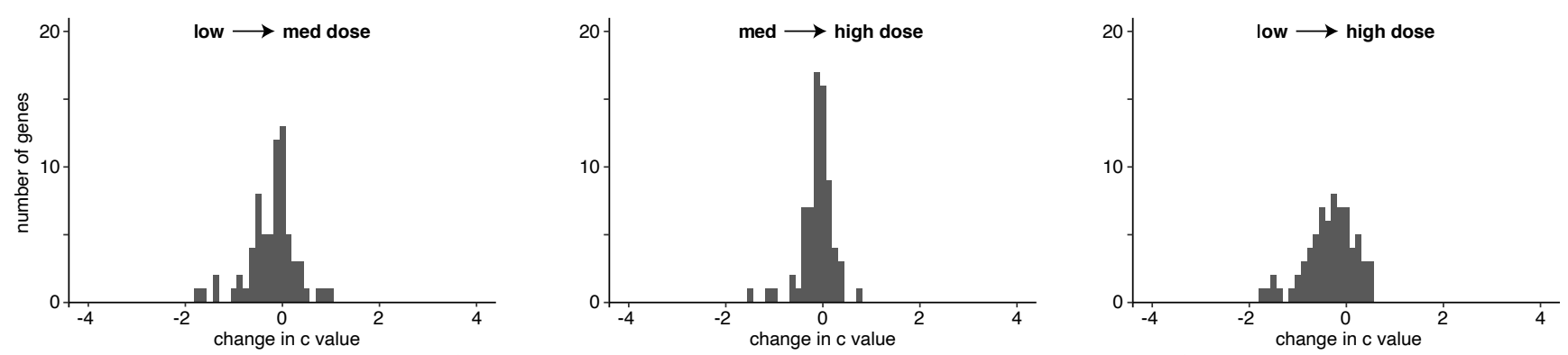

D

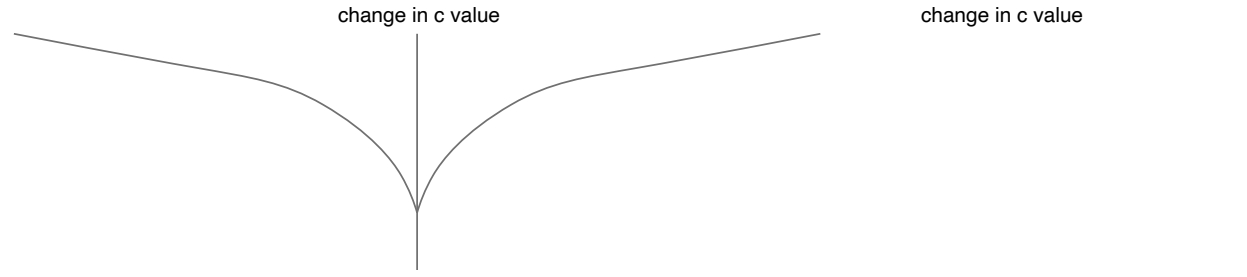

each individual gene in panel C's combined response factor (c value) at low, medium, and high signal dosage

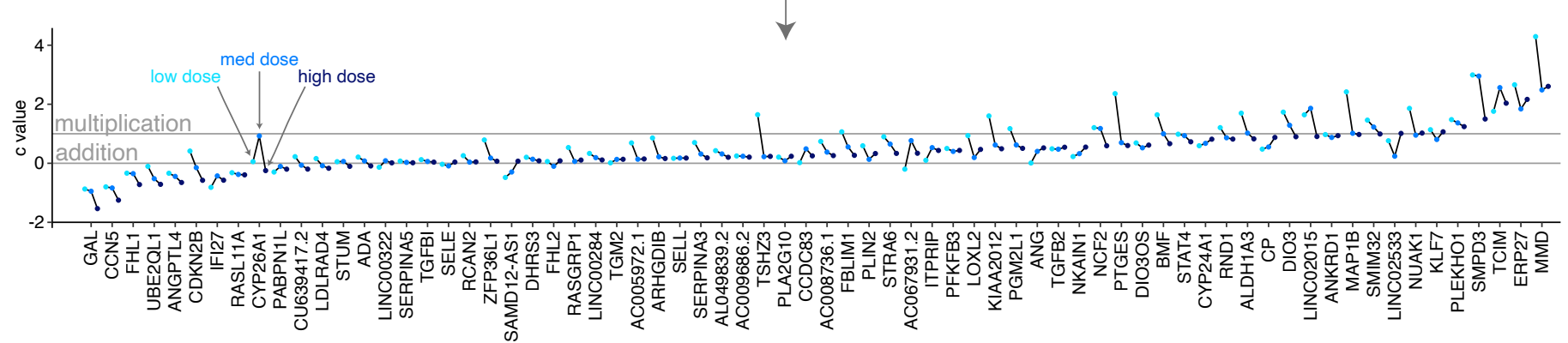




\section{Supplemental Figure 1: The combined response factor tends to remain stable or decrease with increasing signal dosage. A. Mathematical expression showing how we determine the value of the combined response factor (c value) for a given gene and signal dosage. TPM = transcripts per million. B. Histograms illustrating the distribution of $c$ value changes as dosage increases. C. Same histograms as in B, focusing on a subset of genes with stable $\mathrm{c}$ value estimates. D. Sparklines showing the $\mathrm{c}$ value at low, medium, and high dosage for each gene in the subset of genes outlined in panel $\mathrm{C}$.}


bioRxiv preprint doi: https://doi.org/10.1101/2020.05.26.116962; this version posted October 27, 2020. The copyright holder for this preprint (which was not certified by peer review) is the author/funder, who has granted bioRxiv a license to display the preprint in perpetuity. It is made available under aCC-BY 4.0 International license.

\section{Supplemental Figure 2}

A
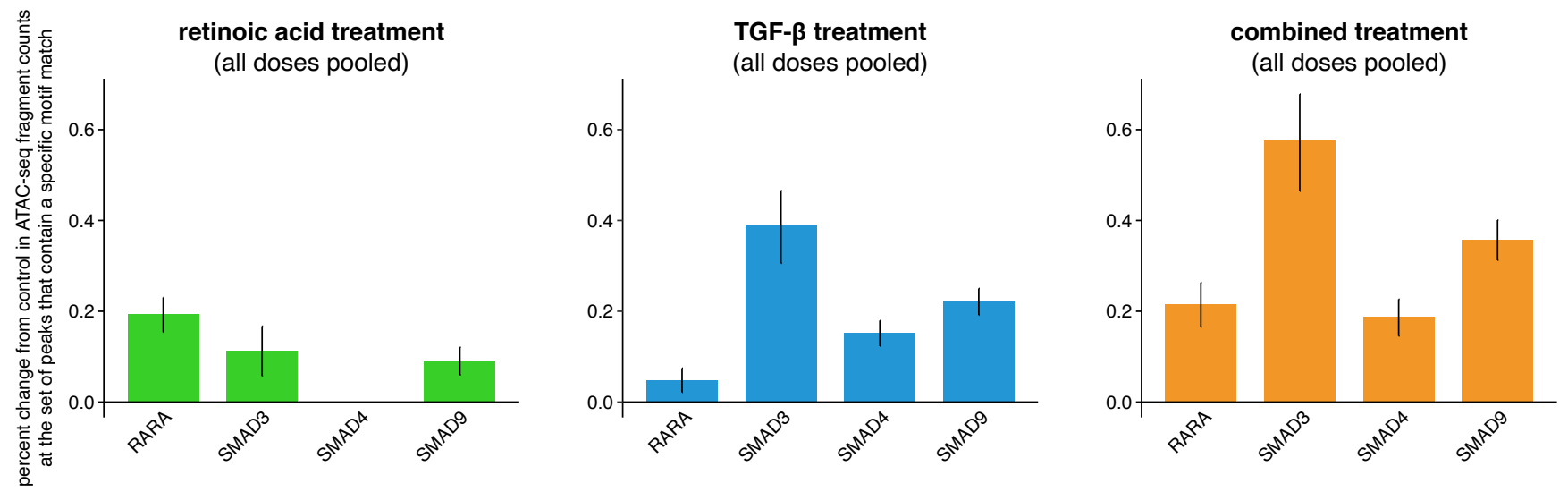

B
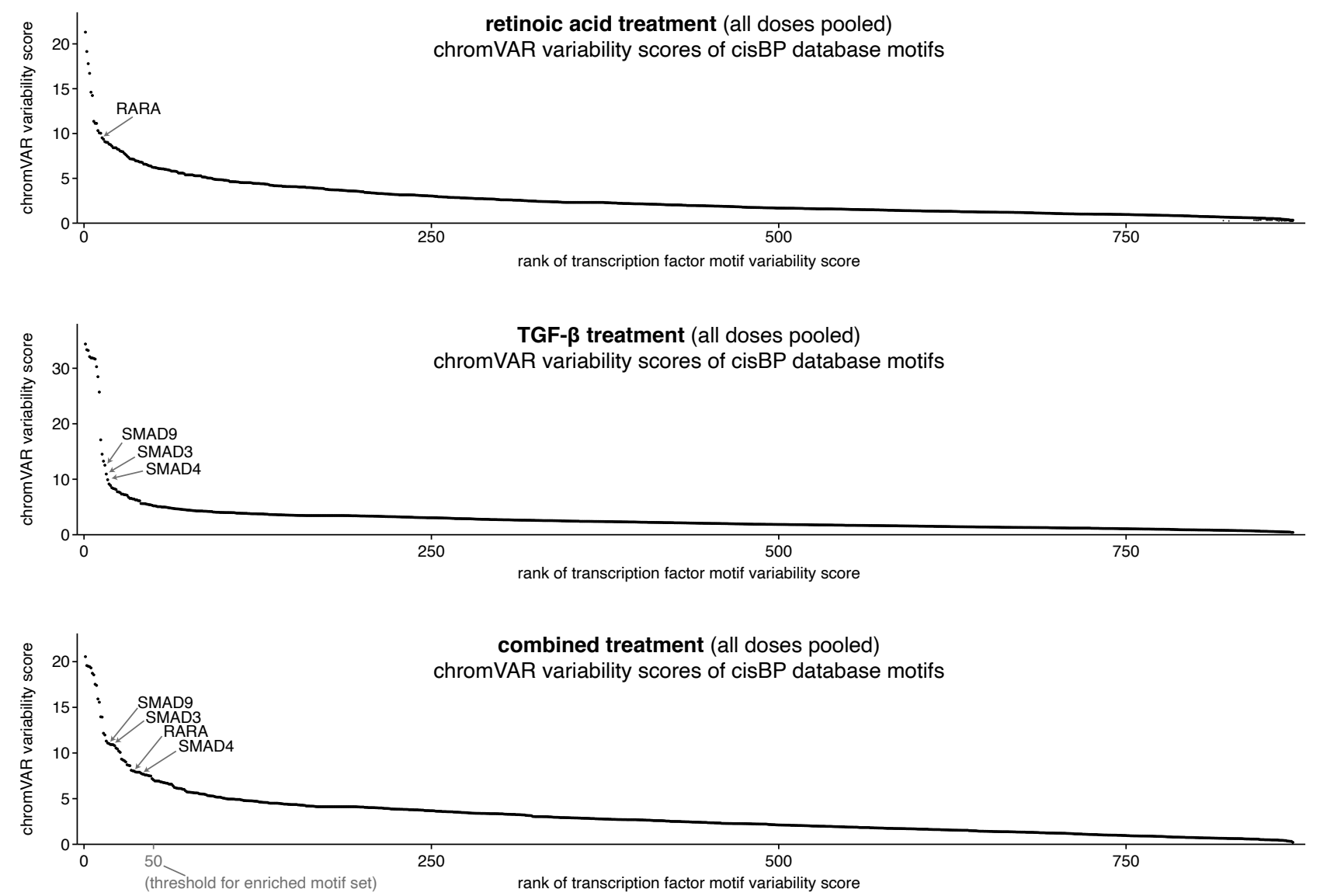


\section{Supplemental Figure 2: Canonical retinoic acid and TGF- $\beta$ signaling motifs (RARA, SMAD3, SMAD4, SMAD9) are enriched in their respective signal treatment conditions. $A$.} Motif enrichment scores for canonical retinoic acid and TGF- $\beta$ effectors. The score reflects the average percent change in ATAC-seq fragment counts compared to ethanol control when a peak contains the given motif. B. chromVAR variability scores (Schep et al. 2017) for all 870 motifs in the curated cisBP motif database for each experimental condition Weirauch et al. 2014). RARA had the 13th highest variability in the retinoic acid condition and SMAD3, SMAD4, and SMAD9 had 17th, 16th, and 14th highest chromVAR variability scores, respectively, in the TGF- $\beta$ condition. For both panels $A$ and $B$, we included all 9 ethanol controls (including low and high cell density controls) and all 9 replicates of each experimental condition (pooling together low, medium, and high dose) when calculating these scores. 
bioRxiv preprint doi: https://doi.org/10.1101/2020.05.26.116962; this version posted October 27, 2020. The copyright holder for this preprint (which was not certified by peer review) is the author/funder, who has granted bioRxiv a license to display the preprint in perpetuity. It is made available under aCC-BY 4.0 International license.

\section{Supplemental Figure 3}

difference between observed ATAC-seq peak counts and additive model prediction
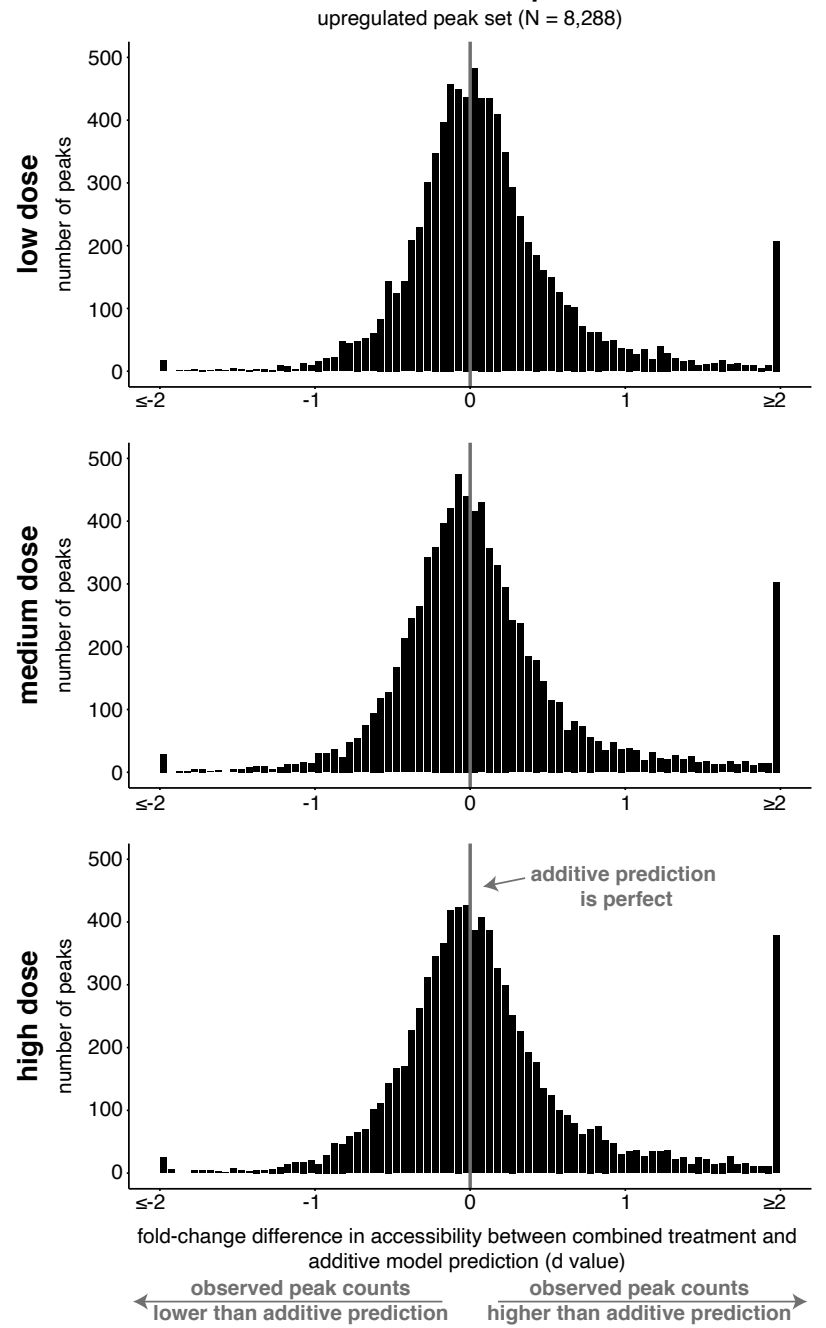

difference between observed ATAC-seq peak counts and multiplicative model prediction
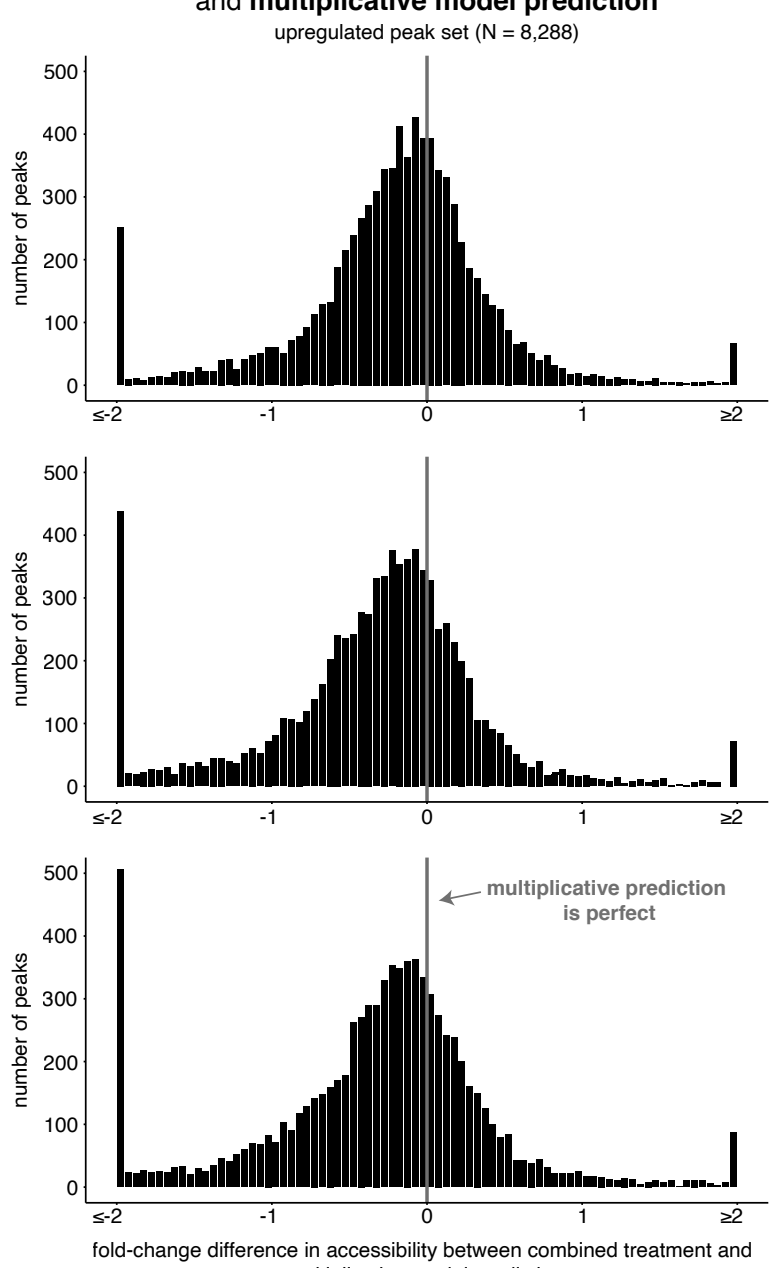
multiplicative model prediction

$\stackrel{\text { observed peak counts }}{\stackrel{\text { lower than mult. prediction }}{n} \quad \frac{\text { observed peak counts }}{\text { higher than mult. prediction }}}$ 


\section{Supplemental Figure 3: The combined response of peaks upregulated individually by retinoic acid and TGF- $\beta$ is more consistent with an additive model than a multiplicative \\ model. For each dose, histograms of the difference between the observed normalized fragment counts at each upregulated peak and their respective additive or multiplicative predictions. The observed differences are centered at zero for the main probability mass for the additive model but not for the multiplicative model.}


bioRxiv preprint doi: https://doi.org/10.1101/2020.05.26.116962; this version posted October 27, 2020. The copyright holder for this preprint (which was not certified by peer review) is the author/funder, who has granted bioRxiv a license to display the preprint in perpetuity. It is made available under aCC-BY 4.0 International license.

\section{Supplemental Figure 4}

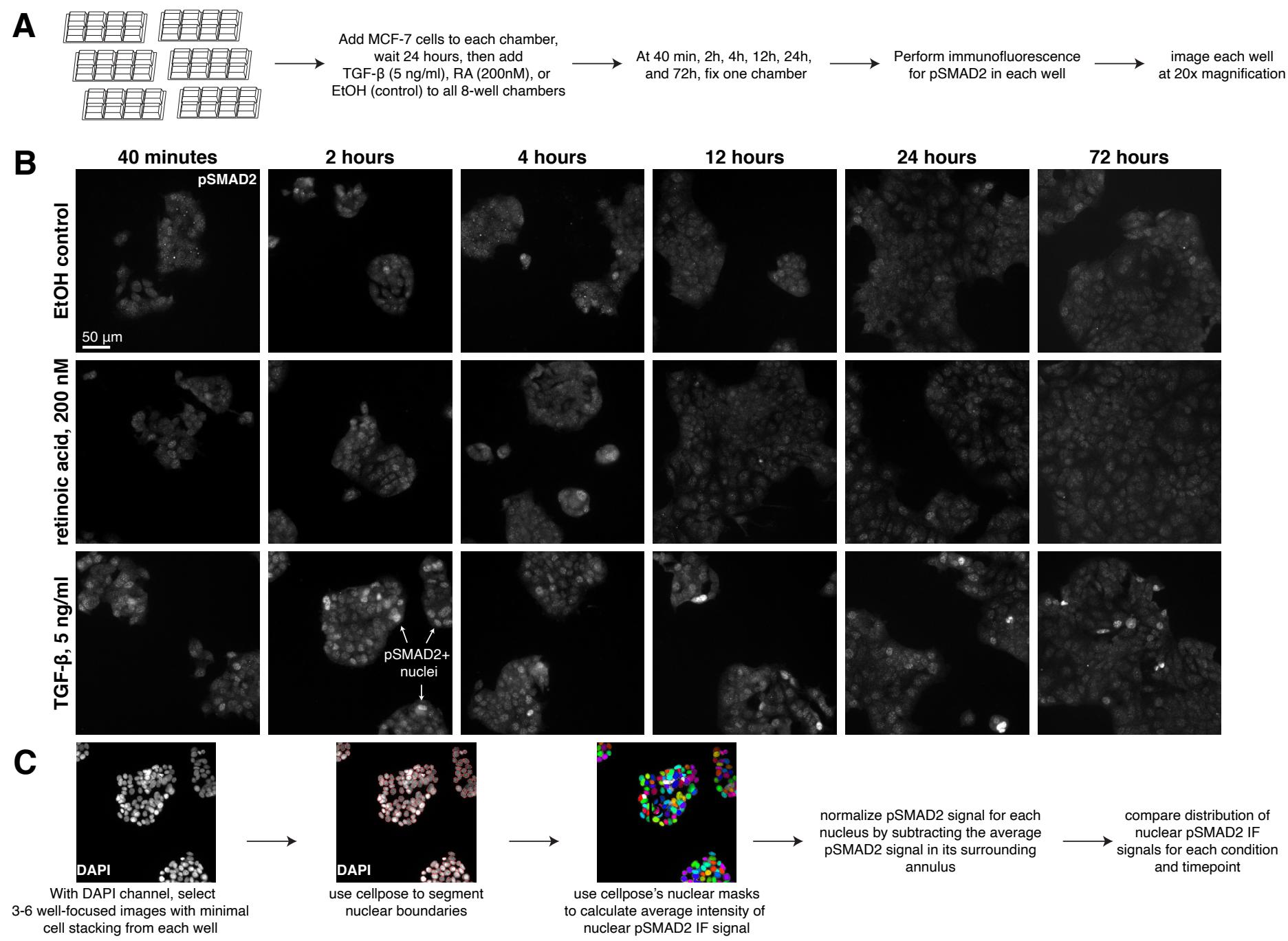

\section{D}

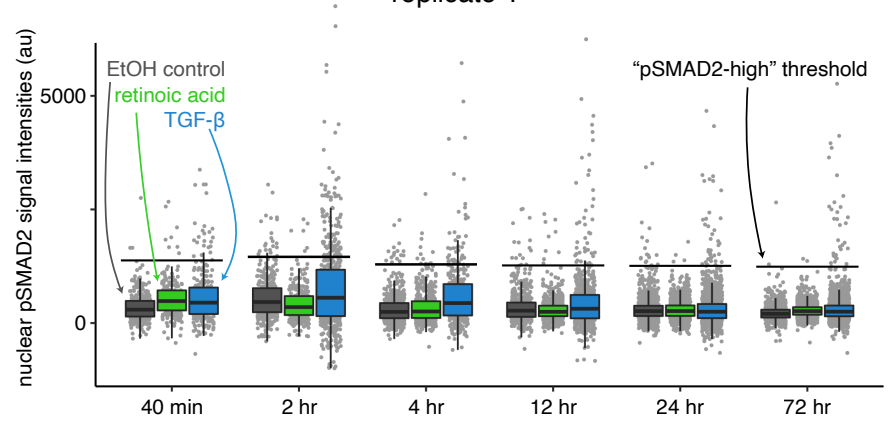

E

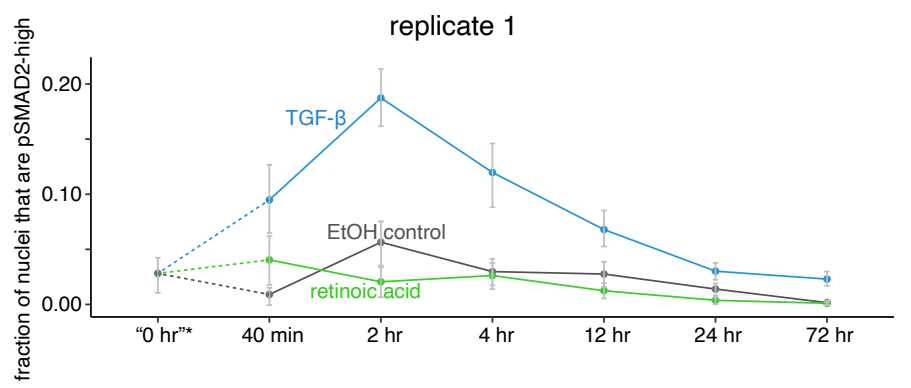

replicate 2
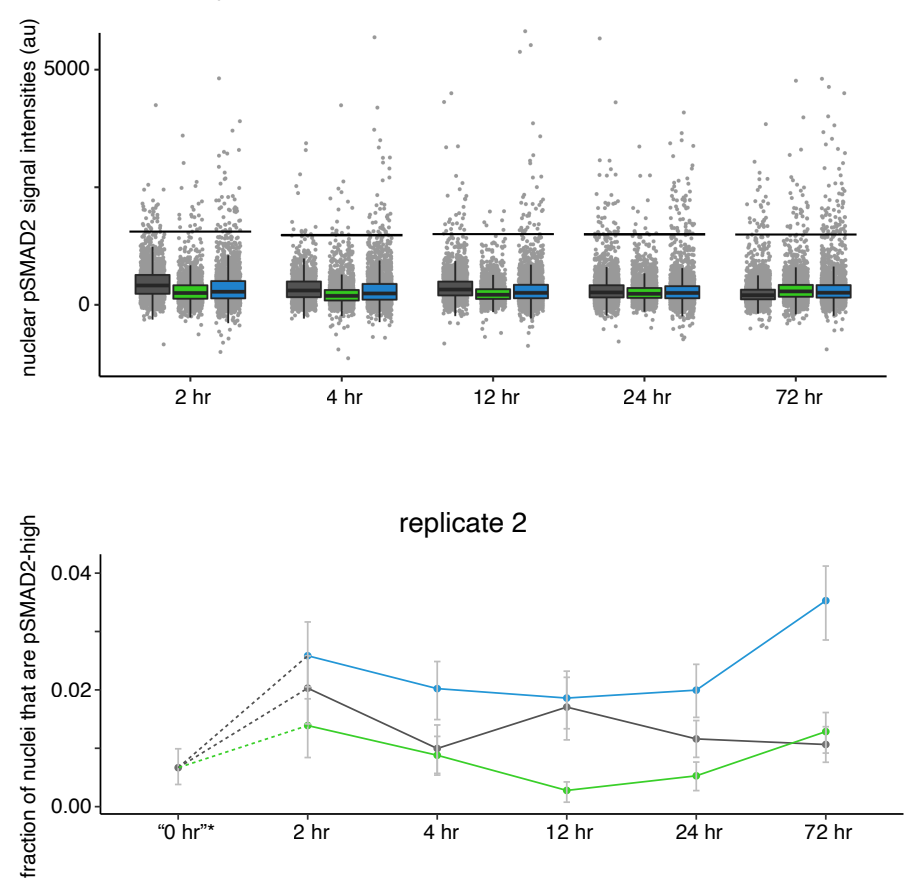


\section{Supplemental Figure 4: TGF-beta, and not retinoic acid, leads to an increase in nuclear pSMAD2 levels in MCF-7 cells. A. Schematic describing immunofluorescence experiment} design. B. Example images of pSMAD2 immunofluorescence for the medium dose of TGF-beta, retinoic acid, or control cells at each time point. C. Computational analysis workflow schematic, which describes how we use the cellpose segmentation algorithm in the DAPI channel to identify nuclear boundaries for measuring average nuclear signal intensity in the pSMAD2 immunofluorescence channel. D. Summary statistics of normalized pSMAD2 nuclear intensity, where each point represents the average immunofluorescence signal intensity of one nucleus. Black horizontal bars used to define a "pSMAD2 high" threshold are 1000 au above the median nuclear intensity value across all conditions in the same time point. E. Fraction of cells that are pSMAD2-high across time for each condition, separated by replicate. *0 hour time point is a pseudo-time point that we generated from additional images taken in the control condition at the 40 minute (replicate 1) or 2 hour time point (replicate 2). 
bioRxiv preprint doi: https://doi.org/10.1101/2020.05.26.116962; this version posted October 27, 2020. The copyright holder for this preprint (which was not certified by peer review) is the author/funder, who has granted bioRxiv a license to display the preprint in perpetuity. It is made available under aCC-BY 4.0 International license.

\section{Supplemental Figure 5}
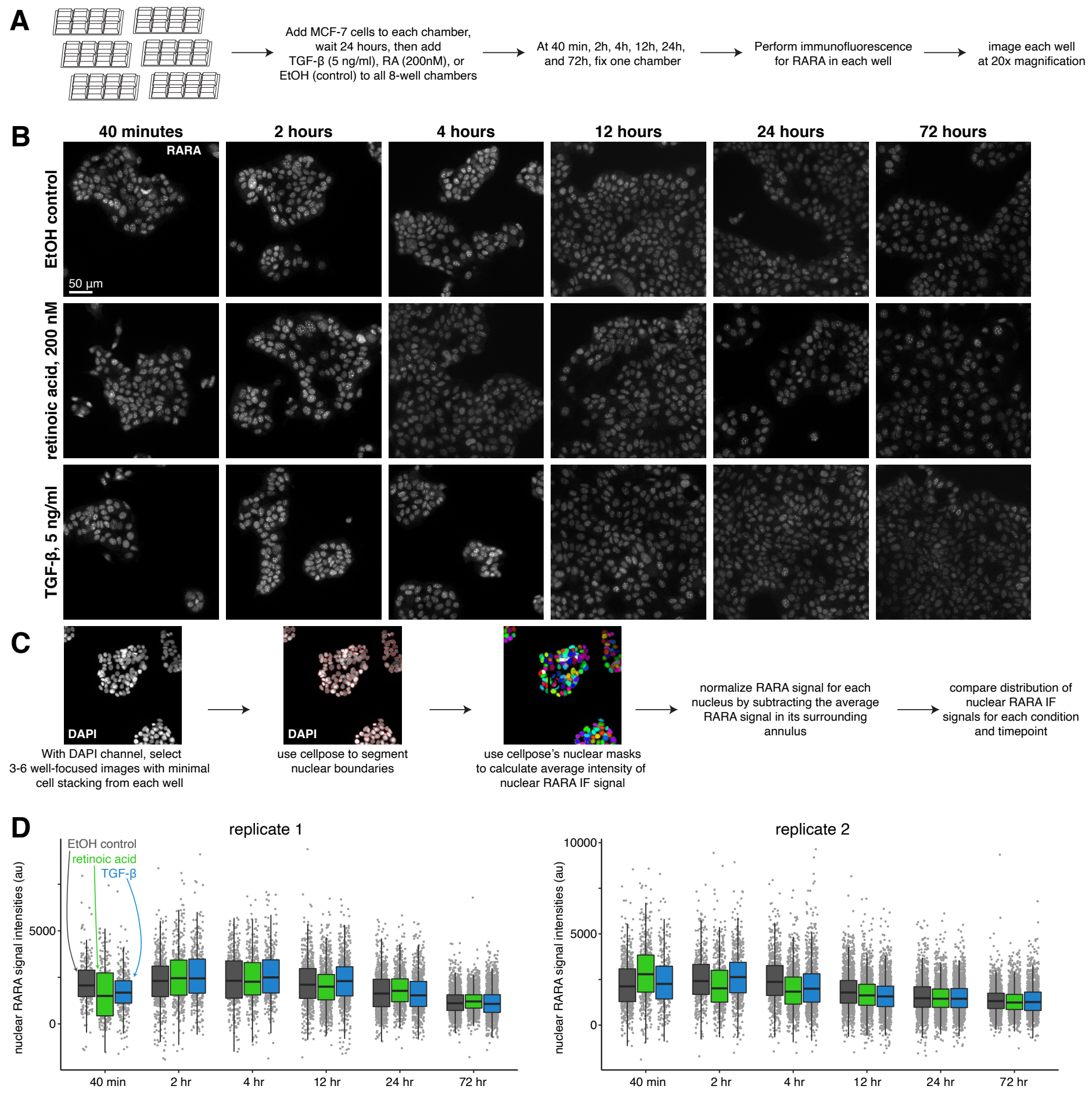


\section{Supplemental Figure 5: Nuclear retinoic acid receptor alpha levels are stable across treatment conditions. A. Schematic describing immunofluorescence experiment design. B. Example images of RARA immunofluorescence for each condition (medium dose of retinoic acid, TGF-beta, or control) at each time point. C. Computational analysis workflow schematic, which describes how we use the cellpose segmentation algorithm in the DAPI channel to identify nuclear boundaries for measuring average nuclear signal intensity in the RARA immunofluorescence channel. D. Summary statistics of normalized RARA nuclear intensity, where each point represents the average immunofluorescence signal intensity of one nucleus.}


bioRxiv preprint doi: https://doi.org/10.1101/2020.05.26.116962; this version posted October 27, 2020. The copyright holder for this preprint (which was not certified by peer review) is the author/funder, who has granted bioRxiv a license to display the preprint in perpetuity. It is made available under aCC-BY 4.0 International license.

\section{Supplemental Figure 6}
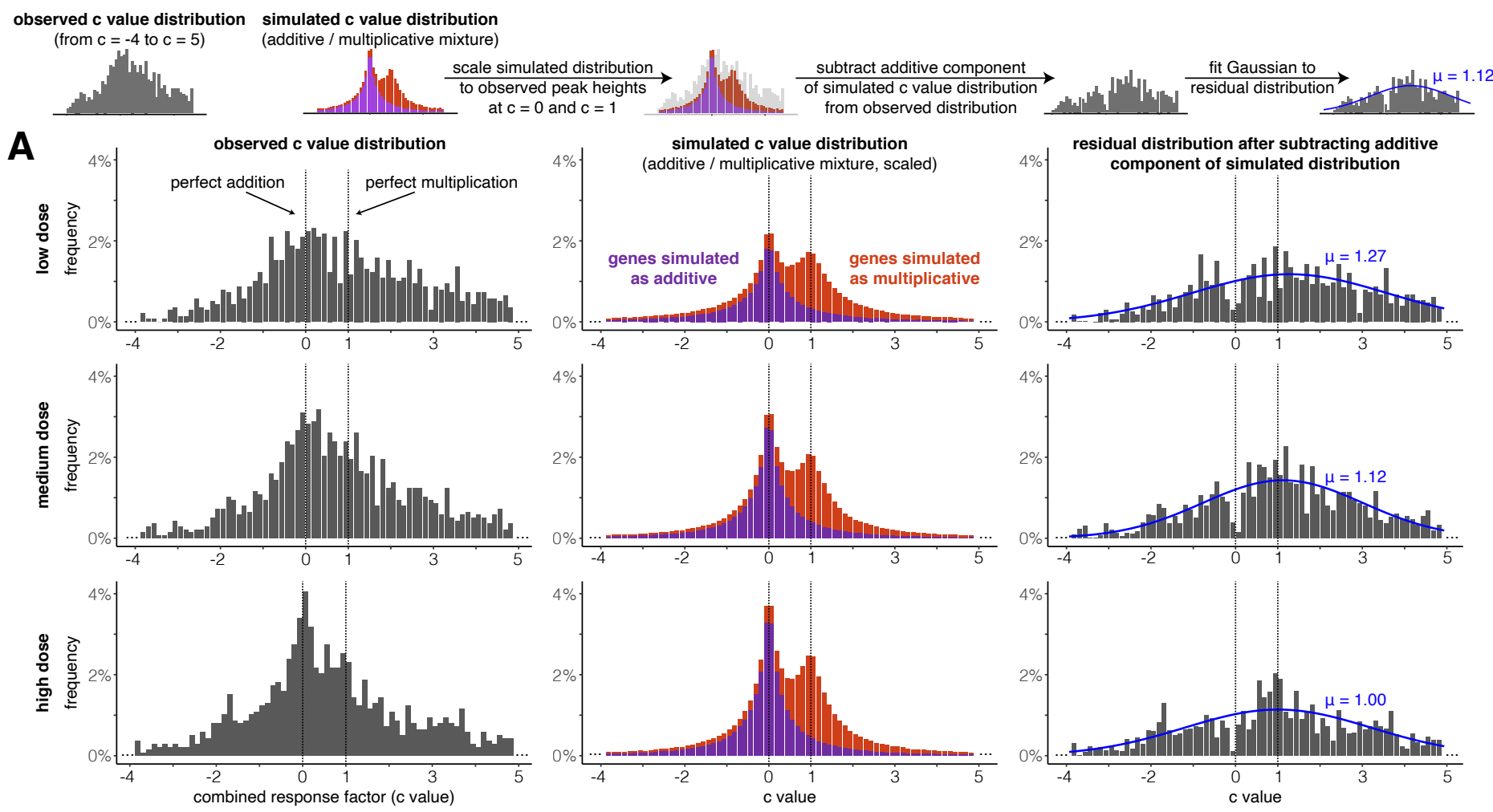

observed $\mathrm{c}$ value distribution simulated $\mathrm{c}$ value distribution
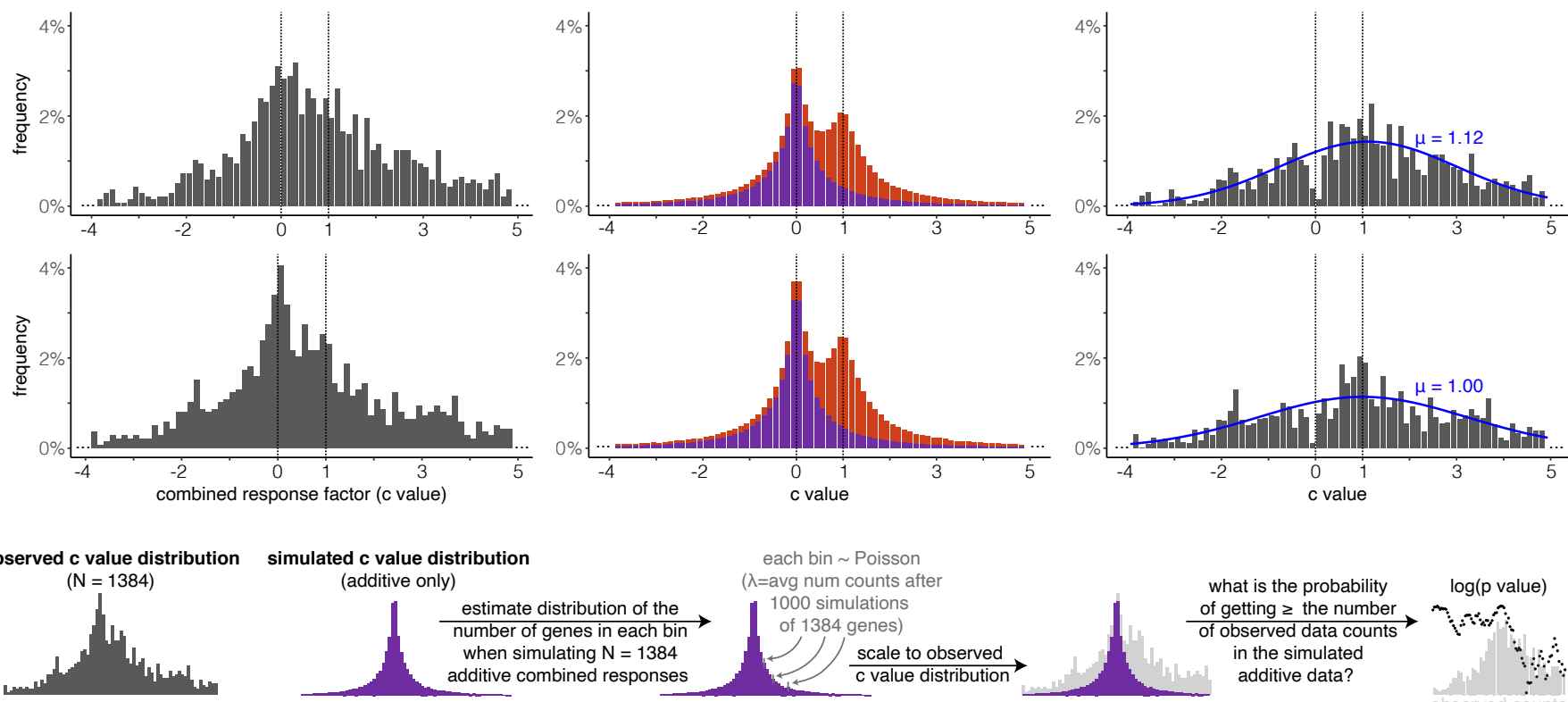

B
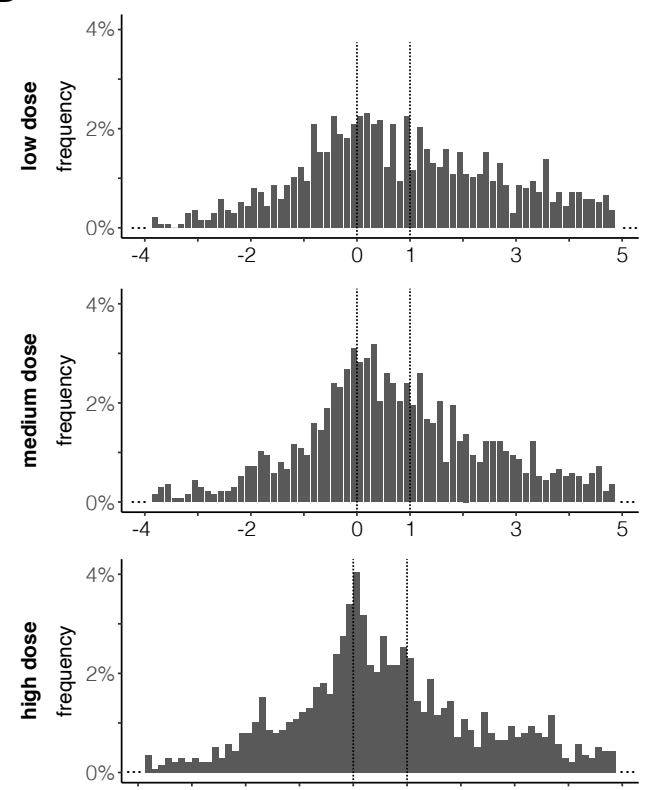

combined response factor (c value) each bin Poisson ( $\lambda=$ avg num counts after $(\lambda=$ avg num counts a 1000 simulations
of 1384 genes) of 1384 genes) $\underset{\text { c value distribution }}{\longrightarrow}$
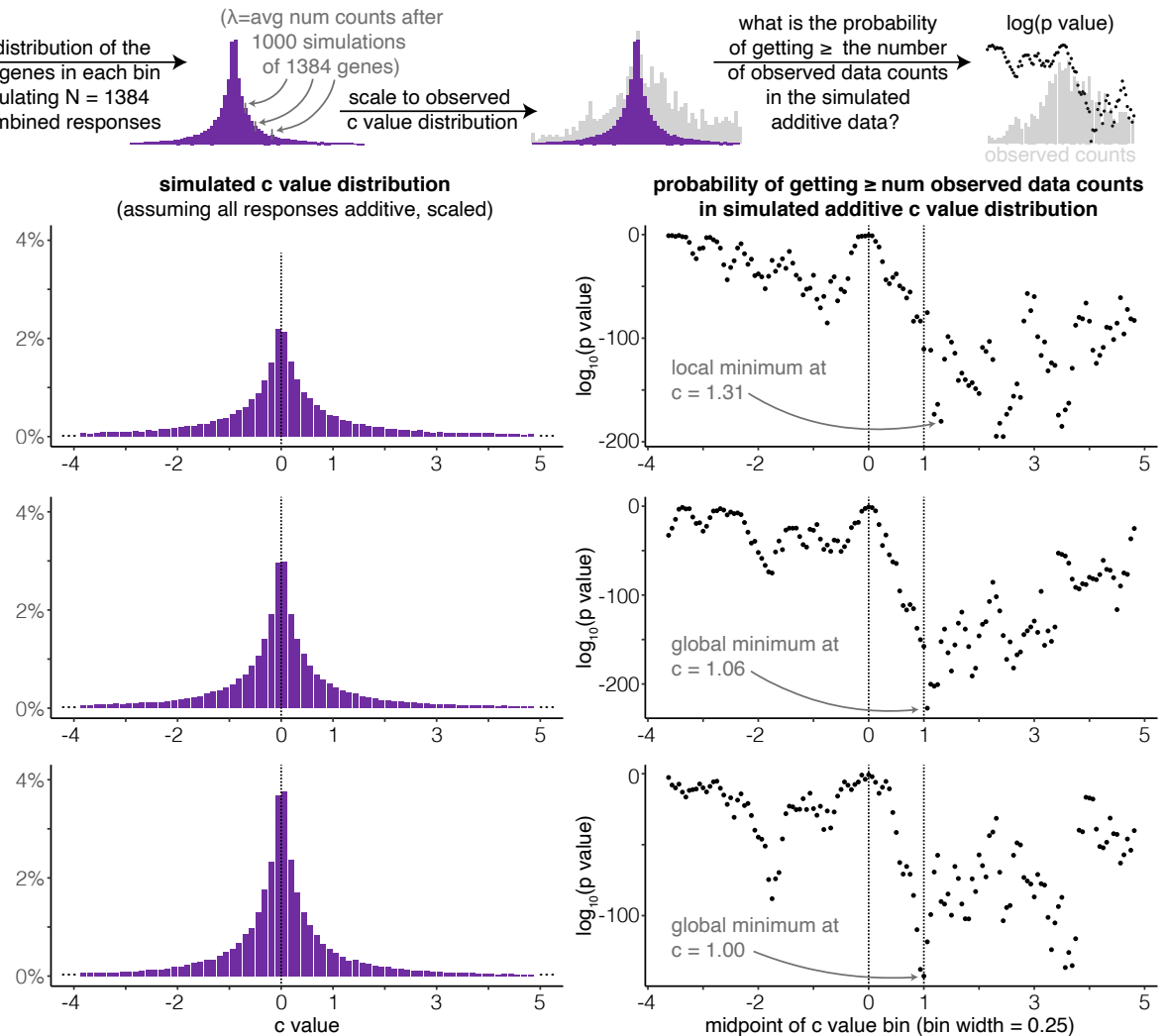

C
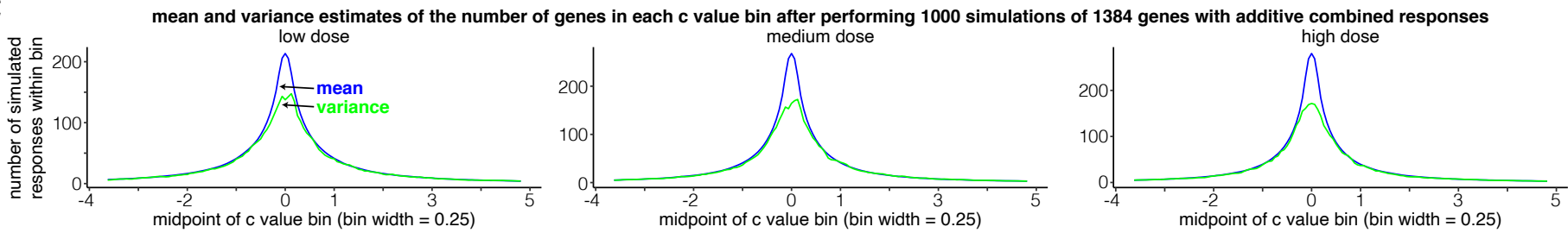


\section{Supplemental Figure 6: A secondary peak occurs at or near perfectly multiplicative combined transcriptional responses $(c=1)$ after subtracting a distribution of simulated additive responses from the observed distribution of $c$ values. $A$. For each dose, the observed $c$ value distribution (in the range from $c=-4$ to $c=5$ ), simulated $c$ value distribution when transcriptional responses are assumed to be either additive or multiplicative, and residual distributions after the additive component of the simulated data is subtracted. Blue lines illustrate the best-fit Gaussian to the residual distribution (see Methods for details). B. At each dose, a c value distribution generated by simulated additive responses is matched to the height of the peak at $c=0$ in the observed data. Then, the probability of witnessing the number of combined responses in each of the bins in the observed histogram is estimated under the null model that all combined responses are additive. Due to the limits of computational power and the extremely low probabilities of witnessing the number of observations as the bins move further away from $c=0$, we assumed Poisson distribution was descriptive of the number of simulated observations that lied in a given $\mathrm{c}$ value bin (this assumption is justified by panel $\mathrm{C}$, where the mean and variance of the number of simulated responses in each bin are nearly identical outside the narrow range of $-0.3<c<0.3$ ). C. At each dose, the mean and variance of the number of simulated additive $c$ values found in each $c$ value bin.}


bioRxiv preprint doi: https://doi.org/10.1101/2020.05.26.116962; this version posted October 27, 2020. The copyright holder for this preprint (which was not certified by peer review) is the author/funder, who has granted bioRxiv a license to display the preprint in perpetuity. It is made available under aCC-BY 4.0 International license.

\section{Supplemental Figure 7}

A

\section{example gene $X$, measurements of} TPM for each condition

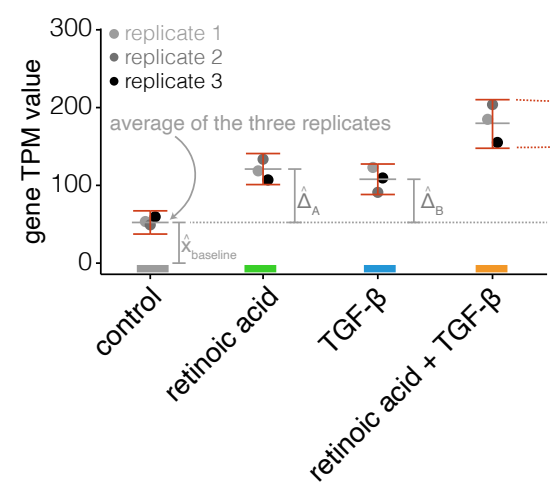

estimated Gaussian distribution underlying measured TPM values for a given condition

B

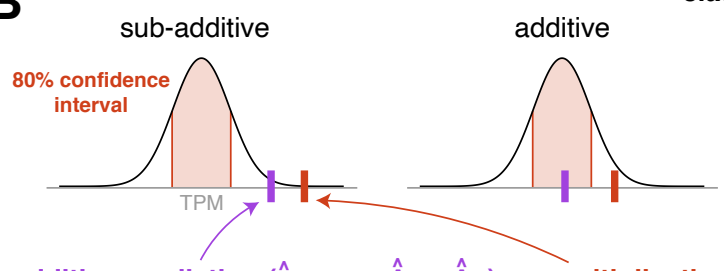

classification of combined responses

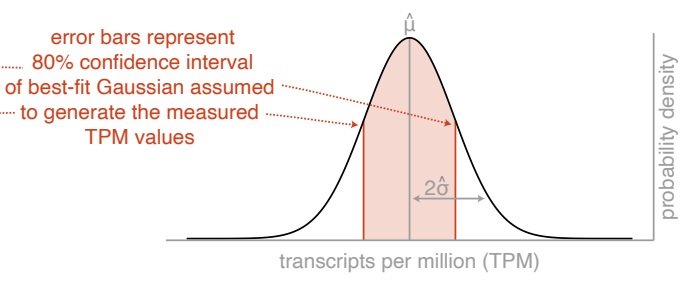

method for estimating mean and variance of Gaussian distribution estimated to underlie each condition

$$
\begin{aligned}
& \hat{\mu}_{\mathrm{s}, \mathrm{d}}=\frac{1}{3} \sum_{\mathrm{r}=1}^{3} \operatorname{TPM}\left(\text { replicate }_{\mathrm{r}}\right) \\
& \hat{\mathrm{\sigma}}_{\mathrm{s}, \mathrm{d}}=\hat{\mu}_{\mathrm{s}, \mathrm{d}} \times \hat{C V}_{\mathrm{s}} \\
& \hat{C V}_{\mathrm{s}}=\frac{1}{3} \sum_{\mathrm{d}=1}^{3} \hat{C V}_{\mathrm{s}, \mathrm{d}} \\
& \hat{C V}_{\mathrm{s}, \mathrm{d}}=\left(1+\frac{1}{4 \mathrm{n}}\right) \frac{\mathrm{s}_{\mathrm{s}, \mathrm{d}}}{\hat{\mu}_{\mathrm{s}, \mathrm{d}}} \quad(\mathrm{n}=3 \text { replicates }) \\
& \mathrm{s}_{\mathrm{s}, \mathrm{d}}=\sqrt{\frac{\sum_{\mathrm{r}=1}^{3}\left(\mathrm{TPM}\left(\text { replicate }_{\mathrm{r}}\right)-\hat{\mu}_{\mathrm{s}, \mathrm{d}}\right)^{2}}{\mathrm{n}-1}} \\
& \begin{array}{l}
\text { subscript definitions: } \\
\text { s indicates signal(s) given } \\
\text { d indicates dosage of the signal(s) given } \\
\mathrm{r} \text { indicates the replicate number }
\end{array}
\end{aligned}
$$

additive prediction $\left(\hat{\mathrm{x}}_{\text {baseline }}+\hat{\Delta}_{A}+\hat{\Delta}_{B}\right) \quad$ multiplicative prediction $\left(\hat{\mathrm{x}}_{\text {baseline }}+\hat{\Delta}_{A}+\hat{\Delta}_{B}+\frac{\hat{\Delta}_{A} \times \hat{\Delta}_{B}}{\hat{x}_{\text {baseline }}}\right)$

C simulating one new set of observations under the assumption that the combined response is additive

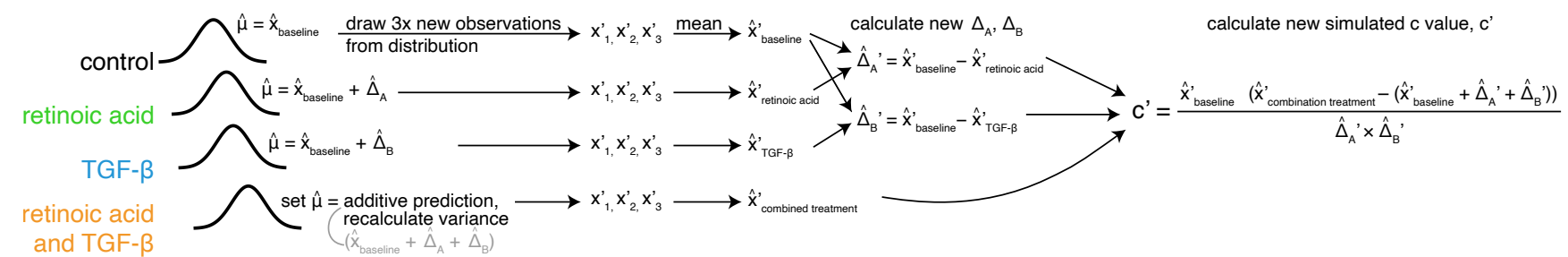

D master set* of upregulated genes (at any dose)
RA distribution of $c$ values when simulated observations are expected to have additive combined responses
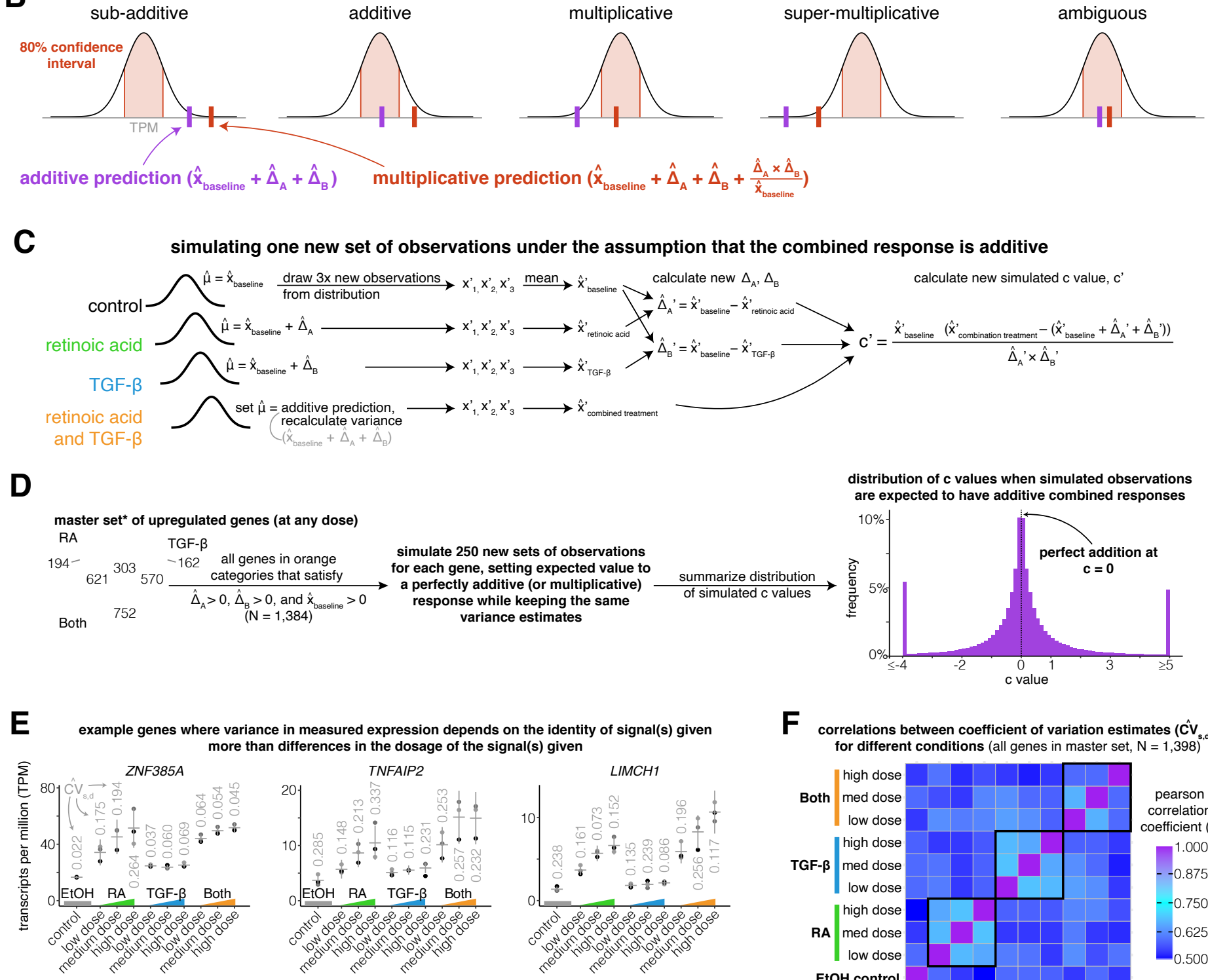

retinoic acid
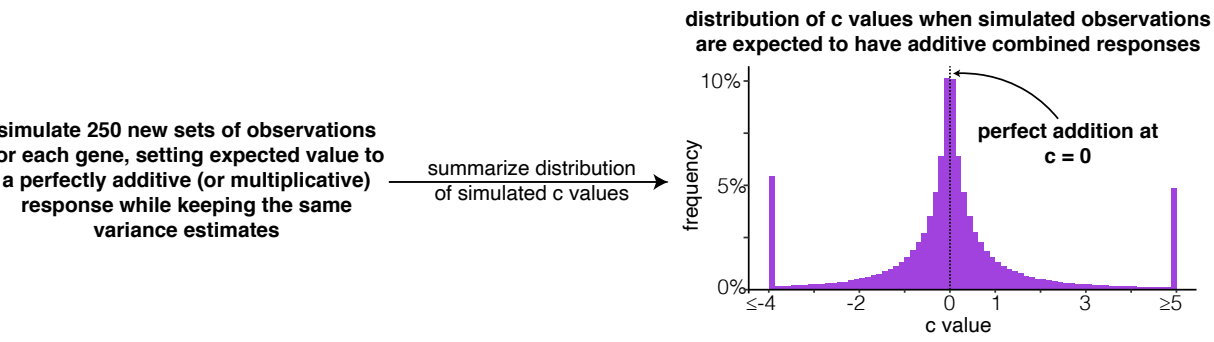

E

re variance in measured expression depends on the identity of signal(s) given differences in the dosage of the signal(s) given
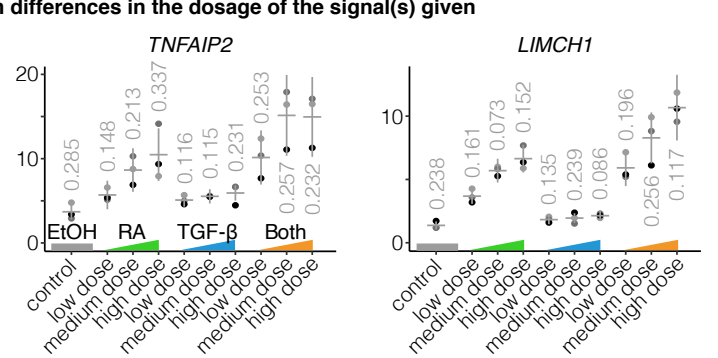

$\mathbf{F}$

- correlations between coefficient of variation estimates $\left(\hat{\mathrm{CV}} \mathrm{s}_{\mathrm{sd}}\right)$ for different conditions (all genes in master set, $\mathrm{N}=1,398$ )
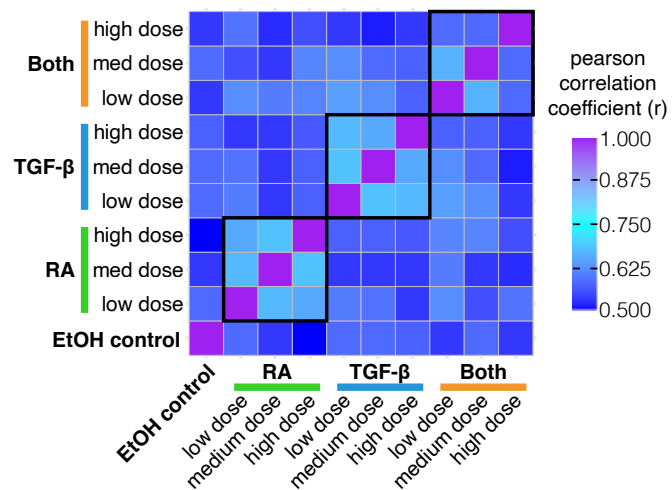


\section{Supplemental Figure 7: Explanatory schematics for model of gene expression variation, classification of combined responses, and simulating new additive or multiplicative} combined responses. A. Schematic illustrating how we estimate the $80 \%$ confidence interval of a Gaussian distribution underlying our gene expression measurements. B. Illustration of how we use the $80 \%$ confidence interval to classify combined responses in Figure $1 \mathrm{E}$ as sub-additive, additive, multiplicative, super-multiplicative, or ambiguous. C. Diagram showing how we simulate new observations by using the Gaussian distributions we estimated to underlie each condition and dose's gene expression measurements. D. Schematic showing how we combine many simulated combined responses from each gene in our master set (less any gene with a control TPM measurement of 0 ) to create a new distribution of simulated $\mathrm{C}$ values. E. Example genes illustrating how the variance of a gene expression measurement often depends on the signal(s) given. F. Correlation matrix showing the Pearson correlation between coefficient of variation (CV) estimates between each condition, with black boxes outlining correlations between different doses of the same signal(s). 


\section{Supplemental Table 1. Post-sequencing ATAC-seq metrics for each sample.}

\begin{tabular}{|c|c|c|c|c|c|c|c|}
\hline Condition & Replicate & $\begin{array}{l}\text { Initial } \\
\text { number of } \\
\text { read pairs } \\
\text { (before } \\
\text { alignment) }\end{array}$ & $\begin{array}{l}\text { Final } \\
\text { number of } \\
\text { read pairs } \\
\text { (aligned, } \\
\text { filtered, } \\
\text { duplicates } \\
\text { removed) }\end{array}$ & $\begin{array}{l}\text { Estimated } \\
\text { library size from } \\
\text { Picard } \\
\text { MarkDuplicates } \\
\text { report }\end{array}$ & $\begin{array}{l}\text { PCR } \\
\text { bottleneck } \\
\text { coefficient }\end{array}$ & $\begin{array}{l}\text { Percent } \\
\text { mitochondrial } \\
\text { reads }\end{array}$ & $\begin{array}{l}\text { Percent of } \\
\text { reads within } \\
500 \text { bp of a } \\
\text { RefSeq } \\
\text { transcription } \\
\text { start site }\end{array}$ \\
\hline EtOH-control & rep1 & $47,581,870$ & $39,444,496$ & $262,895,599$ & 0.94 & $2.8 \%$ & $11.9 \%$ \\
\hline EtOH-control-halfDensity & rep1 & $41,086,622$ & $34,396,506$ & $250,581,572$ & 0.95 & $2.4 \%$ & $11.5 \%$ \\
\hline EtOH-control-highDensity & rep1 & $44,072,790$ & $36,117,206$ & $231,669,308$ & 0.95 & $4.5 \%$ & $11.6 \%$ \\
\hline RA-low-dose & rep1 & $41,260,701$ & $33,591,663$ & $217,146,943$ & 0.95 & $5.3 \%$ & $10.7 \%$ \\
\hline RA-med-dose & rep1 & $31,647,052$ & $26,123,190$ & $179,897,280$ & 0.95 & $5.8 \%$ & $11.0 \%$ \\
\hline RA-high-dose & rep1 & $42,261,199$ & $33,811,647$ & $199,969,660$ & 0.95 & $7.6 \%$ & $11.4 \%$ \\
\hline TGF- $\beta$-low-dose & rep1 & $37,747,257$ & $31,026,429$ & $202,820,143$ & 0.94 & $3.9 \%$ & $10.5 \%$ \\
\hline TGF- $\beta$-med-dose & rep1 & $44,624,493$ & $36,419,357$ & $224,406,039$ & 0.94 & $4.3 \%$ & $10.2 \%$ \\
\hline TGF- $\beta$-high-dose & rep1 & $38,094,133$ & $31,396,736$ & $208,404,962$ & 0.95 & $4.8 \%$ & $10.9 \%$ \\
\hline Both-low-dose & rep1 & $52,387,369$ & $40,479,658$ & $211,802,845$ & 0.95 & $10.1 \%$ & $10.5 \%$ \\
\hline Both-med-dose & rep1 & $49,853,358$ & $39,318,933$ & $225,987,476$ & 0.95 & $8.9 \%$ & $10.5 \%$ \\
\hline Both-high-dose & rep1 & $42,206,009$ & $33,786,922$ & $204,244,183$ & 0.95 & $8.1 \%$ & $10.2 \%$ \\
\hline EtOH-control & rep2 & $38,083,282$ & $31,779,309$ & $226,734,741$ & 0.94 & $2.5 \%$ & $11.5 \%$ \\
\hline EtOH-control-halfDensity & rep2 & $43,361,513$ & $36,593,932$ & $269,862,386$ & 0.94 & $1.5 \%$ & $10.6 \%$ \\
\hline EtOH-control-highDensity & rep2 & $39,698,228$ & $33,195,307$ & $255,254,502$ & 0.95 & $1.8 \%$ & $11.2 \%$ \\
\hline RA-low-dose & rep2 & $36,715,104$ & $30,292,869$ & $207,792,170$ & 0.95 & $4.6 \%$ & $10.8 \%$ \\
\hline RA-med-dose & rep2 & $40,558,424$ & $33,800,414$ & $250,171,599$ & 0.95 & $3.7 \%$ & $10.4 \%$ \\
\hline RA-high-dose & rep2 & $41,414,691$ & $34,358,578$ & $244,841,654$ & 0.95 & $4.0 \%$ & $10.7 \%$ \\
\hline TGF- $\beta$-low-dose & rep2 & $44,817,804$ & $36,658,961$ & $215,298,493$ & 0.93 & $3.4 \%$ & $10.3 \%$ \\
\hline TGF- $\beta$-med-dose & rep2 & $39,929,259$ & $33,087,956$ & $238,242,632$ & 0.95 & $3.5 \%$ & $10.2 \%$ \\
\hline TGF- $\beta$-high-dose & rep2 & $46,205,287$ & $38,202,058$ & $251,952,977$ & 0.94 & $2.8 \%$ & $10.8 \%$ \\
\hline Both-low-dose & rep2 & $40,462,498$ & $33,185,265$ & $250,065,164$ & 0.96 & $5.1 \%$ & $8.8 \%$ \\
\hline Both-med-dose & rep2 & $44,699,468$ & $35,917,746$ & $219,591,584$ & 0.95 & $7.7 \%$ & $9.8 \%$ \\
\hline Both-high-dose & rep2 & $48,925,327$ & $39,315,827$ & $229,690,180$ & 0.94 & $6.1 \%$ & $10.9 \%$ \\
\hline EtOH-control & rep3 & $42,812,546$ & $35,309,638$ & $231,408,229$ & 0.94 & $3.2 \%$ & $12.3 \%$ \\
\hline EtOH-control-halfDensity & rep3 & $41,813,530$ & $34,814,520$ & $258,011,226$ & 0.95 & $3.0 \%$ & $11.6 \%$ \\
\hline EtOH-control-highDensity & rep3 & $40,546,148$ & $33,264,452$ & $204,352,162$ & 0.94 & $4.1 \%$ & $12.5 \%$ \\
\hline RA-low-dose & rep3 & $32,762,330$ & $26,916,331$ & $179,857,516$ & 0.95 & $6.1 \%$ & $11.4 \%$ \\
\hline RA-med-dose & rep3 & $50,878,299$ & $40,363,941$ & $221,908,252$ & 0.94 & $7.4 \%$ & $11.4 \%$ \\
\hline RA-high-dose & rep3 & $44,212,167$ & $33,875,013$ & $165,272,130$ & 0.94 & $10.7 \%$ & $11.7 \%$ \\
\hline TGF- $\beta$-low-dose & rep3 & $45,587,448$ & $37,129,061$ & $231,608,100$ & 0.94 & $4.7 \%$ & $11.3 \%$ \\
\hline TGF- $\beta$-med-dose & rep3 & $38,493,959$ & $31,108,095$ & $182,114,358$ & 0.94 & $5.7 \%$ & $11.1 \%$ \\
\hline
\end{tabular}


bioRxiv preprint doi: https://doi. org/10.1101/2020.05.26.116962; this version posted October 27, 2020. The copyright holder for this preprint (which was not certified by peer review) is the author/funder, who has granted bioRxiv a license to display the preprint in perpetuity. It is made available under aCC-BY 4.0 International license.

\begin{tabular}{|l|c|c|c|c|c|c|c|}
\hline TGF- $\beta$-high-dose & rep3 & $30,124,631$ & $24,795,889$ & $163,300,521$ & 0.94 & $4.7 \%$ & $11.4 \%$ \\
\hline Both-low-dose & rep3 & $50,426,925$ & $38,748,904$ & $196,832,887$ & 0.94 & $10.3 \%$ & $10.9 \%$ \\
\hline Both-med-dose & rep3 & $44,423,642$ & $34,365,274$ & $183,838,974$ & 0.95 & $11.0 \%$ & $10.8 \%$ \\
\hline Both-high-dose & rep3 & $42,164,691$ & $32,326,317$ & $169,428,244$ & 0.95 & $11.2 \%$ & $10.6 \%$ \\
\hline
\end{tabular}




\section{Acknowledgments}

We thank John Murray, Ken Zaret, Golnaz Vahedi, Nancy Zhang, Caroline Bartman, and Yogesh Goyal for helpful ideas and conversations, Connie Jiang, Chris Coté and Ryan Boe for assistance with figure design, Connie Jiang for assistance with cell culture, Amanpreet Kaur, Naveen Jain, and lan Dardani for assistance with immunofluorescence and associated imaging, Lauren Beck and Phil Burnham for assistance with immunofluorescence image analysis, and Caroline Bartman and Yogesh Goyal for critical review of the manuscript. EMS acknowledges support from NIH training grant F30 HG010986, BLE acknowledges support from NIH training grants F30 CA236129, T32 GM007170 and T32 HG000046, AJC acknowledges support from $\mathrm{NIH}$ training grant T32 GM-07229, and AR acknowledges support from R01 CA238237, NIH Director's Transformative Research Award R01 GM137425, R01 CA232256, NSF CAREER 1350601, P30 CA016520, SPORE P50 CA174523, NIH U01 CA227550, NIH 4DN U01 HL129998, NIH Center for Photogenomics (RM1 HG007743), and the Tara Miller Foundation.

\section{Author contributions}

AR and EMS conceived the study and designed experiments. EMS conducted all experiments and performed all data analyses. BLE supervised EMS during cell culture, RNA-seq library preparation, and ATAC-seq library preparation. EMS, AR, BLE, and AC wrote the paper.

\section{Competing interests}

AR receives royalties related to Stellaris RNA FISH probes. All other authors declare no competing interests. 


\section{References}

Ackers GK, Johnson AD, Shea MA. 1982. Quantitative model for gene regulation by lambda phage repressor. Proc Natl Acad Sci U S A 79:1129-1133.

Bartman CR, Hamagami N, Keller CA, Giardine B, Hardison RC, Blobel GA, Raj A. 2019. Transcriptional Burst Initiation and Polymerase Pause Release Are Key Control Points of Transcriptional Regulation. Mol Cell 73:519-532.e4.

Bender MA, Ragoczy T, Lee J, Byron R, Telling A, Dean A, Groudine M. 2012. The hypersensitive sites of the murine $\beta$-globin locus control region act independently to affect nuclear localization and transcriptional elongation. Blood 119:3820-3827.

Bintu L, Buchler NE, Garcia HG, Gerland U, Hwa T, Kondev J, Phillips R. 2005. Transcriptional regulation by the numbers: models. Curr Opin Genet Dev 15:116-124.

Blau J, Xiao H, McCracken S, O'Hare P, Greenblatt J, Bentley D. 1996. Three functional classes of transcriptional activation domain. Mol Cell Biol 16:2044-2055.

Bogard N, Linder J, Rosenberg AB, Seelig G. 2019. A Deep Neural Network for Predicting and Engineering Alternative Polyadenylation. Cell 178:91-106.e23.

Bothma JP, Garcia HG, Ng S, Perry MW, Gregor T, Levine M. 2015. Enhancer additivity and non-additivity are determined by enhancer strength in the Drosophila embryo. Elife 4. doi:10.7554/eLife.07956

Buenrostro JD, Giresi PG, Zaba LC, Chang HY, Greenleaf WJ. 2013. Transposition of native chromatin for fast and sensitive epigenomic profiling of open chromatin, DNA-binding proteins and nucleosome position. Nat Methods 10:1213-1218.

Corces MR, Trevino AE, Hamilton EG, Greenside PG, Sinnott-Armstrong NA, Vesuna S, Satpathy AT, Rubin AJ, Montine KS, Wu B, Kathiria A, Cho SW, Mumbach MR, Carter AC, Kasowski M, Orloff LA, Risca VI, Kundaje A, Khavari PA, Montine TJ, Greenleaf WJ, Chang HY. 2017. Omni-ATAC-seq: Improved ATAC-seq protocol. doi:10.1038/protex.2017.096

Cunliffe HE, Ringnér M, Bilke S, Walker RL, Cheung JM, Chen Y, Meltzer PS. 2003. The gene expression response of breast cancer to growth regulators: patterns and correlation with tumor expression profiles. Cancer Res 63:7158-7166.

Fiore C, Cohen BA. 2016. Interactions between pluripotency factors specify cis-regulation in embryonic stem cells. Genome Res 26:778-786.

Fuda NJ, Ardehali MB, Lis JT. 2009. Defining mechanisms that regulate RNA polymerase II transcription in vivo. Nature 461:186-192.

Fulco CP, Nasser J, Jones TR, Munson G, Bergman DT, Subramanian V, Grossman SR, Anyoha R, Doughty BR, Patwardhan TA, Nguyen TH, Kane M, Perez EM, Durand NC, Lareau CA, Stamenova EK, Aiden EL, Lander ES, Engreitz JM. 2019. Activity-by-contact model of enhancer-promoter regulation from thousands of CRISPR perturbations. Nat Genet 51:1664-1669.

Gertz J, Siggia ED, Cohen BA. 2009. Analysis of combinatorial cis-regulation in synthetic and genomic promoters. Nature 457:215-218.

Geva-Zatorsky N, Dekel E, Cohen AA, Danon T, Cohen L, Alon U. 2010. Protein dynamics in drug combinations: a linear superposition of individual-drug responses. Cell 140:643-651.

Ginley-Hidinger M, Carleton JB, Rodriguez AC, Berrett KC, Gertz J. 2019. Sufficiency analysis of estrogen responsive enhancers using synthetic activators. Life Sci Alliance 2. doi:10.26508/lsa.201900497

Goldstein I, Paakinaho V, Baek S, Sung M-H, Hager GL. 2017. Synergistic gene expression during the acute phase response is characterized by transcription factor assisted loading. Nat Commun 8:1849. 
Gotea V, Visel A, Westlund JM, Nobrega MA, Pennacchio LA, Ovcharenko I. 2010. Homotypic clusters of transcription factor binding sites are a key component of human promoters and enhancers. Genome Res 20:565-577.

Hua S, Kittler R, White KP. 2009. Genomic antagonism between retinoic acid and estrogen signaling in breast cancer. Cell 137:1259-1271.

Jin F, Li Y, Dixon JR, Selvaraj S, Ye Z, Lee AY, Yen C-A, Schmitt AD, Espinoza CA, Ren B. 2013. A high-resolution map of the three-dimensional chromatin interactome in human cells. Nature 503:290-294.

Joung JK, Koepp DM, Hochschild A. 1994. Synergistic activation of transcription by bacteriophage lambda cl protein and E. coli cAMP receptor protein. Science 265:1863-1866.

Kaplan S, Bren A, Zaslaver A, Dekel E, Alon U. 2008. Diverse two-dimensional input functions control bacterial sugar genes. Mol Cell 29:786-792.

Klemm SL, Shipony Z, Greenleaf WJ. 2019. Chromatin accessibility and the regulatory epigenome. Nat Rev Genet. doi:10.1038/s41576-018-0089-8

Kwasnieski JC, Fiore C, Chaudhari HG, Cohen BA. 2014. High-throughput functional testing of ENCODE segmentation predictions. Genome Res 24:1595-1602.

Love MI, Huber W, Anders S. 2014. Moderated estimation of fold change and dispersion for RNA-seq data with DESeq2. Genome Biol 15:550.

Mahdi SHA, Cheng H, Li J, Feng R. 2015. The effect of TGF-beta-induced epithelial-mesenchymal transition on the expression of intracellular calcium-handling proteins in T47D and MCF-7 human breast cancer cells. Arch Biochem Biophys 583:18-26.

Mangelsdorf DJ, Evans RM. 1995. The RXR heterodimers and orphan receptors. Cell 83:841-850.

Napoli JL. 1986. [13] Quantification of physiological levels of retinoic acidMethods in Enzymology. Academic Press. pp. 112-124.

Nechaev S, Adelman K. 2011. Pol II waiting in the starting gates: Regulating the transition from transcription initiation into productive elongation. Biochim Biophys Acta 1809:34-45.

Noman MZ, Janji B, Abdou A, Hasmim M, Terry S, Tan TZ, Mami-Chouaib F, Thiery JP, Chouaib S. 2017. The immune checkpoint ligand PD-L1 is upregulated in EMT-activated human breast cancer cells by a mechanism involving ZEB-1 and miR-200. Oncoimmunology 6:e1263412.

Patwardhan RP, Hiatt JB, Witten DM, Kim MJ, Smith RP, May D, Lee C, Andrie JM, Lee S-I, Cooper GM, Ahituv N, Pennacchio LA, Shendure J. 2012. Massively parallel functional dissection of mammalian enhancers in vivo. Nat Biotechnol 30:265-270.

Phillips R, Belliveau NM, Chure G, Garcia HG, Razo-Mejia M, Scholes C. 2019. Figure 1 Theory Meets Figure 2 Experiments in the Study of Gene Expression. Annu Rev Biophys 48:121-163.

Rao SSP, Huntley MH, Durand NC, Stamenova EK, Bochkov ID, Robinson JT, Sanborn AL, Machol I, Omer AD, Lander ES, Aiden EL. 2014. A 3D map of the human genome at kilobase resolution reveals principles of chromatin looping. Cell 159:1665-1680.

Rapakoulia T, Gao X, Huang Y, de Hoon M, Okada-Hatakeyama M, Suzuki H, Arner E. 2017. Genome-scale regression analysis reveals a linear relationship for promoters and enhancers after combinatorial drug treatment. Bioinformatics 33:3696-3700.

Rosenberg AB, Patwardhan RP, Shendure J, Seelig G. 2015. Learning the sequence determinants of alternative splicing from millions of random sequences. Cell 163:698-711.

Rothschild D, Dekel E, Hausser J, Bren A, Aidelberg G, Szekely P, Alon U. 2014. Linear 
superposition and prediction of bacterial promoter activity dynamics in complex conditions. PLoS Comput Biol 10:e1003602.

Ruf S, Symmons O, Uslu VV, Dolle D, Hot C, Ettwiller L, Spitz F. 2011. Large-scale analysis of the regulatory architecture of the mouse genome with a transposon-associated sensor. Nat Genet 43:379-386.

Schep AN, Wu B, Buenrostro JD, Greenleaf WJ. 2017. chromVAR: inferring transcription-factor-associated accessibility from single-cell epigenomic data. Nat Methods 14:975-978.

Scholes C, Biette KM, Harden TT, DePace AH. 2019. Signal Integration by Shadow Enhancers and Enhancer Duplications Varies across the Drosophila Embryo. Cell Rep 26:2407-2418.e5.

Scholes C, DePace AH, Sánchez Á. 2017. Combinatorial Gene Regulation through Kinetic Control of the Transcription Cycle. Cell Syst 4:97-108.e9.

Sherman MS, Cohen BA. 2012. Thermodynamic state ensemble models of cis-regulation. PLoS Comput Biol 8:e1002407.

Stampfel G, Kazmar T, Frank O, Wienerroither S, Reiter F, Stark A. 2015. Transcriptional regulators form diverse groups with context-dependent regulatory functions. Nature 528:147-151.

Stringer C, Wang T, Michaelos M, Pachitariu M. 2020. Cellpose: a generalist algorithm for cellular segmentation. doi:10.1101/2020.02.02.931238

Thurman RE, Rynes E, Humbert R, Vierstra J, Maurano MT, Haugen E, Sheffield NC, Stergachis AB, Wang H, Vernot B, Garg K, John S, Sandstrom R, Bates D, Boatman L, Canfield TK, Diegel M, Dunn D, Ebersol AK, Frum T, Giste E, Johnson AK, Johnson EM, Kutyavin T, Lajoie B, Lee B-K, Lee K, London D, Lotakis D, Neph S, Neri F, Nguyen ED, Qu H, Reynolds AP, Roach V, Safi A, Sanchez ME, Sanyal A, Shafer A, Simon JM, Song L, Vong S, Weaver M, Yan Y, Zhang Z, Zhang Z, Lenhard B, Tewari M, Dorschner MO, Hansen RS, Navas PA, Stamatoyannopoulos G, lyer VR, Lieb JD, Sunyaev SR, Akey JM, Sabo PJ, Kaul R, Furey TS, Dekker J, Crawford GE, Stamatoyannopoulos JA. 2012. The accessible chromatin landscape of the human genome. Nature 489:75-82.

Tian M, Schiemann WP. 2017. TGF- $\beta$ Stimulation of EMT Programs Elicits Non-genomic ER- $a$ Activity and Anti-estrogen Resistance in Breast Cancer Cells. J Cancer Metastasis Treat 3:150-160.

Weirauch MT, Yang A, Albu M, Cote AG, Montenegro-Montero A, Drewe P, Najafabadi HS, Lambert SA, Mann I, Cook K, Zheng H, Goity A, van Bakel H, Lozano J-C, Galli M, Lewsey MG, Huang E, Mukherjee T, Chen X, Reece-Hoyes JS, Govindarajan S, Shaulsky G, Walhout AJM, Bouget F-Y, Ratsch G, Larrondo LF, Ecker JR, Hughes TR. 2014. Determination and inference of eukaryotic transcription factor sequence specificity. Cell 158:1431-1443.

Yang Y, Fear J, Hu J, Haecker I, Zhou L, Renne R, Bloom D, Mclntyre LM. 2014. Leveraging biological replicates to improve analysis in ChIP-seq experiments. Comput Struct Biotechnol J 9:e201401002.

Zaret KS, Carroll JS. 2011. Pioneer transcription factors: establishing competence for gene expression. Genes Dev 25:2227-2241.

Zhang C, Tu H-L, Jia G, Mukhtar T, Taylor V, Rzhetsky A, Tay S. 2019. Ultra-multiplexed analysis of single-cell dynamics reveals logic rules in differentiation. Sci Adv 5:eaav7959. 